\title{
Repetitive Model of Mild Traumatic Brain Injury Produces Cortical Abnormalities Detectable by Magnetic Resonance Diffusion Imaging, Histopathology, and Behavior
}

\author{
Fengshan Yu,,2 Dinesh K. Shukla, ${ }^{1,5}$ Regina C. Armstrong, ${ }^{1-3}$ Christina M. Marion, ${ }^{1,3}$ \\ Kryslaine L. Radomski, ${ }^{1,2}$ Reed G. Selwyn, ${ }^{1,6,{ }^{*}}$ and Bernard J. Dardzinski, ${ }^{1,4,{ }^{*}}$
}

\begin{abstract}
Noninvasive detection of mild traumatic brain injury (mTBI) is important for evaluating acute through chronic effects of head injuries, particularly after repetitive impacts. To better detect abnormalities from mTBI, we performed longitudinal studies (baseline, 3, 6, and 42 days) using magnetic resonance diffusion tensor imaging (DTI) and diffusion kurtosis imaging $(\mathrm{DKI})$ in adult mice after repetitive $\mathrm{mTBI}(\mathrm{r}-\mathrm{mTBI}$; daily $\times 5)$ or sham procedure. This $\mathrm{r}-\mathrm{mTBI}$ produced righting reflex delay and was first characterized in the corpus callosum to demonstrate low levels of axon damage, astrogliosis, and microglial activation, without microhemorrhages. High-resolution DTI-DKI was then combined with post-imaging pathological validation along with behavioral assessments targeted for the impact regions. In the corpus callosum, only DTI fractional anisotropy at 42 days showed significant change post-injury. Conversely, cortical regions under the impact site (M1-M2, anterior cingulate) had reduced axial diffusivity (AD) at all time points with a corresponding increase in axial kurtosis $\left(\mathrm{K}_{\mathrm{a}}\right)$ at 6 days. Post-imaging neuropathology showed microglial activation in both the corpus callosum and cortex at 42 days after r-mTBI. Increased cortical microglial activation correlated with decreased cortical AD after r-mTBI $(r=-0.853 ; n=5)$. Using Thy1-YFP-16 mice to fluorescently label neuronal cell bodies and processes revealed low levels of axon damage in the cortex after r-mTBI. Finally, r-mTBI produced social deficits consistent with the function of this anterior cingulate region of cortex. Overall, vulnerability of cortical regions is demonstrated after mild repetitive injury, with underlying differences of DTI and DKI, microglial activation, and behavioral deficits.
\end{abstract}

Keywords: concussion; diffusion kurtosis imaging; diffusion tensor imaging; microglia; Thy1-YFP

\section{Introduction}

$\mathbf{C}$ ONCUSSION, OR MILD TRAUMATIC BRAIN INJURY (mTBI), is a blow or jolt to the head resulting in brief alteration in consciousness or post-traumatic amnesia lasting less than $24 \mathrm{~h}$, loss of consciousness lasting less than $30 \mathrm{~min}$, and a Glasgow Coma Score between 13 and $15 .{ }^{1}$ This type of head impact is common in contact sports, falls, motor vehicle accidents, and as a consequence of blast exposure. mTBI is the most common form of TBI, comprising over $82 \%$ of the 339,462 brain injuries sustained by military personnel between the years 2000 and $2015 .^{2}$ Global incidence of mTBI may be upward of 40 million events per year. ${ }^{3}$ Although most individuals suffering from mTBI recover over a course of 8-12 weeks, approximately $15 \%$ of patients continue to experience symptoms, such as headache, dizziness, insomnia, and difficulties with balance, for much longer. Indeed, over $22 \%$ of mTBI patients were still functionally impaired at 12 months post-injury in a recent prospective study. ${ }^{4}$ Currently available diagnostic tools are not sufficient to accurately predict which patients may suffer persistent symptoms from mTBI. Further, it is critical to determine how repetitive concussive and even subconcussive impacts, that is, below the threshold for mTBI diagnosis, contribute to the long-term consequences of brain injury.

${ }^{1}$ Center for Neuroscience and Regenerative Medicine, ${ }^{2}$ Department of Anatomy, Physiology and Genetics, ${ }^{3}$ Program in Neuroscience, and ${ }^{4}$ Department of Radiology and Radiological Sciences, Uniformed Services University of the Health Sciences, Bethesda, Maryland.

${ }^{5}$ Department of Psychiatry, University of Maryland School of Medicine, Baltimore, Maryland.

${ }^{6}$ Department of Radiology, University of New Mexico, Albuquerque, New Mexico.

*R.G.S. and B.J.D. are co-senior authors.

(c) Fengshan Yu, et al., 2017; Published by Mary Ann Liebert, Inc. This Open Access article is distributed under the terms of the Creative Commons Attribution Noncommercial License (http://creativecommons.org/licenses/by-nc/4.0/) which permits any noncommercial use, distribution, and reproduction in any medium, provided the original author(s) and the source are credited. 
Noninvasive modalities, such as neuroimaging techniques, have the potential to detect abnormalities in tissue microstructure and could be implemented for longitudinal studies of injury progression to better determine how early abnormalities predict long-term outcomes.

Conventional neuroimaging modalities, such as computed tomography (CT) and standard sequences for magnetic resonance imaging (MRI), do not detect mTBI. However, nuclear medicine imaging and advanced MRI techniques, such as hypercapnia/blood oxygen level dependent or diffusion tensor imaging (DTI), have shown promise in determining prognosis post-mTBI. ${ }^{5-9}$ To date, pre-clinical and clinical studies using DTI demonstrate both lower and higher fractional anisotropy (FA) in white matter, which indicates loss of structural integrity post-injury. ${ }^{10-13}$ FA values depend on fiber topography among brain regions and the time post-injury, for example, acute, subacute, or chronic stage. ${ }^{14-17}$ FA changes in relationship to changes in diffusivity parallel (axial) and transverse (radial) to fibers. These parameters are influenced by complex signal generated by multiple tissue features, including axon internal elements, myelination, cellularity, and extracellular elements. ${ }^{14,} 18$ Increased FA early post-injury may occur with reduced radial diffusivity, which has been attributed to decreased water content in myelin or extracellular space, ${ }^{19}$ or with increased axial diffusivity, which may be associated with increased fiber track density or gliosis. ${ }^{14}$ Several studies have observed reduced FA in white matter in patients post-mTBI, which, in experimental models, has corresponded with demyelination and axon damage. ${ }^{10-14,18,20}$ Advancing DTI for clinical diagnosis and as a predictive outcome measure will require extended longitudinal studies and a comprehensive understanding of the correlation between abnormal tissue components and imaging results. ${ }^{21}$ Clinical studies can be informed by neuropathology-neuroimaging correlations derived from studies in animal models in which imaging is followed by analysis of tissue microstructure at the cellular level by post-imaging neuropathology.

Advanced diffusion techniques applicable to this purpose include DTI as well as emerging methods to extend the information obtained from restricted and nonrestricted tissue compartments, such as diffusion kurtosis imaging (DKI) or diffusion spectrum imaging. ${ }^{22}$ DTI has several advantages over conventional MRI methods given that it acquires data in multiple directions and uses a tensor to quantitate diffusion in an anisotropic system. Other advantages of DTI include analysis of full brain volume, its ability to allow in vivo tractography of white matter pathways, and its freedom from operator bias. The limitation of DTI is that a linear decay of signal intensity is assumed with increasing diffusion weighting, which measures sensitivity to water diffusion. However, at high diffusion sensitization, the signal attenuation is nonlinear and thus better estimated by accounting for nonGaussian diffusion or kurtosis. Clinical studies evaluating mTBI patients found DKI to be sensitive for detecting abnormalities and associated with cognitive status. ${ }^{23-25}$ DKI has the potential to detect changes in both white and gray matter from acute through subacute changes post-injury, based on studies in experimental TBI using a mild form of the controlled cortical impact (CCI) model in adult rats. ${ }^{26}$ DTI has been used to detect changes in white and gray matter of patients with persistent post-concussive symptoms, ${ }^{27}$ and, recently, DKI was used to detect abnormalities associated with subconcussive impacts in high school football players. ${ }^{28}$

The aim of this study was to characterize changes in this combination of advanced diffusion imaging techniques during the progression of repetitive mTBI (r-mTBI). To this end, we extended the use of DTI and DKI longitudinally from a pre-injury baseline through 6 weeks post-injury followed by post-imaging analysis of tissue pa- thology. We characterize a model of r-mTBI using five attenuated impacts over bregma. Although DTI is typically not considered sensitive to cortical damage, attributed to the low anisotropy of gray matter, the cerebral cortex overlying the corpus callosum has a relatively high density of myelinated fibers associated with motor pathways at the coronal level of bregma. In addition, DKI is expected to be more sensitive to tissue complexity, such as cortical regions. Therefore, the regions of interest (ROIs) in our longitudinal DTI and DKI studies were analyzed for the corpus callosum and for the adjacent medial cerebral cortex. The immunohistochemical analysis of pathology in the corpus callosum was complemented by additional examination of the cortex in Thy1-YFP-16 mice, which have a high density of neurons expressing yellow fluorescent protein (YFP) throughout the cell body, axon, and dendritic arbor. Further, potential functional correlates of the DTI and DKI changes were evaluated using behavioral assessments targeted to the corpus callosum and the adjacent cortical regions within the imaging ROIs.

We show that the combination of DTI and DKI reveals important differences that add to the interpretation of the underlying pathology. Longitudinal studies of a given region across multiple time points aids in detection of abnormalities. Importantly, DTI and DKI changes in the cortical gray matter are associated with microglial activation and later-stage behavioral deficits resulting from repetitive traumatic brain injury.

\section{Methods}

\section{Repetitive mild traumatic brain injury model}

All animals were treated in accord with guidelines of the Uniformed Services University of the Health Sciences and the National Institutes of Health Guide for the Care and Use of Laboratory Animals. Seven-week-old male C57BL/6 mice or Thy1-YFP-16 mice, both from The Jackson Laboratory (Bar Harbor, ME), were housed in standard cages in a 12-h/12-h light-dark cycle with food and water ad libitum before sham or TBI procedures at 8 weeks of age. As a positive control for tau pathology, P301S mice expressing a mutant form of human tau were obtained from Tony Wyss-Coray (Stanford University, Palo Alto, CA) and used at 5 months of age when tau pathology develops in cortical regions. ${ }^{29}$

The parameters of the r-mTBI model were based on pilot testing of multiple protocols (data not shown). An Impact One Stereotaxic Impactor (Leica, Wetzlar, Germany) was used to produce r-mTBI concussive impacts. A series of five impacts separated by $24-\mathrm{h}$ intervals was chosen to target the period of decreased glucose uptake observed $24 \mathrm{~h}$ after a single mTBI in rats. ${ }^{30}$ Mice were anesthetized with $2.0 \%$ isoflurane in $\mathrm{O}_{2}$ and then the hair was shaved and depilated with Nair. Mice were positioned in a stereotaxic frame with rubber stoppers inserted between the external ear canal and ear bar. Impacts were made onto the skin approximately over bregma using a 3-mm-diameter tip (velocity set at $4.0 \mathrm{~m} / \mathrm{sec}$; depth of $1.0 \mathrm{~mm}$; dwell time of $200 \mathrm{~ms}$ ). Sham mice underwent identical procedures to the r-mTBI mice without receiving impacts. Body temperature was maintained with a warming pad. After each procedure, the duration of apnea and the righting reflex were recorded (Fig. 1). The repetitive injury study included $30 \mathrm{r}$-mTBI mice and $30 \mathrm{r}$-sham mice. A single impact (s-mTBI) model has been previously characterized $^{31}$ and is included as supplementary data (Supplementary Fig. 1) (see online supplementary material at http://www.liebertpub .com) for direct comparison of assessment techniques with the novel r-mTBI model through 6 weeks post-injury.

\section{Magnetic resonance image acquisition parameters}

In vivo MRI was performed on a 7 Tesla small animal $(20-\mathrm{cm}$ bore) Bruker BioSpec scanner equipped with $12-\mathrm{cm}$ diameter 
$650 \mathrm{mT} / \mathrm{m}$ gradient coils (Bruker BioSpin $\mathrm{GmbH}$, Reinstetten, Germany). Longitudinal scanning was used to evaluate changes in both the corpus callosum and the overlying cortical gray matter. All mice underwent pre-injury diffusion- and $\mathrm{T}_{2^{-}}$weighted MR imaging and follow-up imaging at 3 days, 6 days, and 6 weeks postinjury. Mice were scanned as yoked pairs consisting of an mTBI mouse with a sham mouse. Two independent cohorts of 3 r-mTBI mice and 3 repeptitive sham ( $\mathrm{r}$-sham) mice were run from surgery through analysis for a total of $6 \mathrm{r}$-mTBI mice and $6 \mathrm{r}$-sham mice scanned at all time points.

Animals were anaesthetized with $1 \%$ isoflurane and placed in an MR compatible mouse bed. Images were acquired with a fourchannel phased array MR coil after volumetric radiofrequency excitation. A two-dimensional (2D) single-shot echo planar imaging sequence (repetition time/echo time $[\mathrm{TR} / \mathrm{TE}]=4500 / 30 \mathrm{msec}$; four repetitions) was used to acquire four unweighted $\left(b=0 \mathrm{~s} / \mathrm{mm}^{2}\right)$ images and seven diffusion-weighted images $(b=400,800,1200,1600,2000$, 2400 , and $2800 \mathrm{~s} / \mathrm{mm}^{2}$ ) using a Stejskal-Tanner diffusion preparation $^{32}$ with parameters of $\Delta=12 \mathrm{msec}, \delta=5 \mathrm{msec}$, field of view $(\mathrm{FOV})=14 \times 14 \mathrm{~mm}^{2}$, matrix $=80 \times 80$, slice thickness $=0.75 \mathrm{~mm}, 18$ slices, and 30 noncollinear diffusion gradient directions. A multiecho 2D rapid acquisition with relaxation enhancement (RARE) sequence $^{33}$ was used to acquire $T_{2}$-weighted images $(T R=4500 \mathrm{msec}$, $\mathrm{TE}=20,60,100$, and $140 \mathrm{msec}$, RARE factor $2, \mathrm{FOV}=14 \times 14 \mathrm{~mm}^{2}$, matrix $=140 \times 140 \times 18$ for s-mTBI and TE $=10,30,50,70,90,110$, and $130 \mathrm{msec}$ and matrix $=100 \times 100 \times 18$ for $\mathrm{r}-\mathrm{mTBI}$, with slice thickness $=0.75 \mathrm{~mm}$ ). Body temperature was maintained at $36^{\circ} \mathrm{C}$ by circulating hot water. Respiration and heart rate were monitored throughout each 2-h imaging session.

\section{Magnetic resonance image analysis}

Each ROI for analysis was manually drawn on five consecutive coronal slices of the non-diffusion-weighted $\left(b=0 \mathrm{~s} / \mathrm{mm}^{2}\right)$ images (one rostral to bregma, one at the coronal level of bregma, and three caudal to bregma). A single-voxel width was used for the corpus callosum ROI. ROIs in the medial cerebral cortex were drawn in both hemispheres superior to the corpus callosum, while keeping one voxel distance of separation from the corpus callosum and cortical surface to avoid partial volume effects. The peak of the cingulum was used to delineate the border of the medial cortical ROIs. Multiple ROIs were drawn on test scans for standardization before analysis of the study data sets. The individual drawing the ROI for the quantitative analysis was blinded to the animal condition.

Diffusion data were analyzed using TORTOISE software. ${ }^{34}$ An average non-diffusion-weighted image $\left(b=0 \mathrm{~s} / \mathrm{mm}^{2}\right.$ image $)$ and all seven diffusion-weighted images were used in the analysis. Diffusion weighted imaging artifacts, including motion, eddy current, and concomitant field distortions, were calculated and combined to enable the image correction to be applied in a single step. This process was used to minimize interpolation errors in the corrected data. Fractional anisotropy (FA) and mean (MD), axial (AD), and radial $(\mathrm{RD})$ diffusivity maps were calculated by the linear least squares method of tensor estimation. Diffusion direction-encoded color (DEC) maps output from the TOROTOISE software were used to demonstrate fiber orientation in the corpus callosum and cortical regions. ${ }^{35}$ Kurtosis data were pre-processed with TORTOISE, and each voxel was analyzed using in-house-developed software in MATLAB (The MathWorks, Inc., Natick, MA) according to a previously published method provided by University of Maryland. Essentially, this software routine fits the logarithm of the normalized signal intensity versus the b-values using linear least squares to a quadratic function. ${ }^{26}$ The signal-to-noise ratio (SNR) of the diffusion-weighted images was $>10$ for $b=2800$ and $>50$ for $\mathrm{b}=0 \mathrm{~s} / \mathrm{mm}^{2}$ images, respectively, where SNR was calculated as: $[(0.655 \times$ signal $) /$ standard deviation of an ROI in the background of the image)]. The average ROI (corpus callosum and cortex) signal was a factor of 3 greater than the average background signal for $b=2800 \mathrm{~s} / \mathrm{mm}^{2}$ and 14 for $b=0 \mathrm{~s} / \mathrm{mm}^{2}$. Quadratic fits to the raw data were robust for the ROIs interrogated with adjusted $R^{2}>0.99$. The mean kurtosis (MK) was calculated by averaging the apparent kurtosis $\left(\mathrm{K}_{\mathrm{app}}\right)$ in 30 directions: $M K=\frac{1}{30} \sum_{i=1}^{30}\left(K_{\text {app }}\right) i$

Axial kurtosis $\left(\mathrm{K}_{\mathrm{a}}\right)$ and radial kurtosis $\left(\mathrm{K}_{\mathrm{r}}\right)$ maps, which characterize the kurtosis along the axial and radial diffusion directions, respectively, were calculated from the three eigenvalues derived from the diffusion kurtosis tensor as described by Jensen and colleagues. ${ }^{36}$ The corpus callosum and medial cortical ROIs were overlaid onto DTI and DKI maps for further quantitative analysis.

\section{Tissue analysis}

Mice were perfused with $4 \%$ paraformaldehyde. Brains were further post-fixed by immersion in the same fixative overnight, cryopreserved, and then cut as coronal sections $(14 \mu \mathrm{m})$. Prussian blue staining combined with pararosaniline nuclear stain (SigmaAldrich, St. Louis, MO) was used to detect hemosiderin-laden macrophages indicative of previous microbleeds.

Immunohistochemical analysis was used to assess axon damage based on $\beta$-amyloid precursor protein $\left(\beta\right.$-APP) ${ }^{37}$ (polyclonal rabbit anti- $\beta$-APP antibody; 1:100; Life Technologies, Grand Island, NY). Microglia/macrophages were detected based on CD11b (monoclonal rat anti-CD11b; 1:100; Abcam, Cambridge, MA). Astrocytes were evaluated by immunostaining for glial fibrillary acidic protein (GFAP; polyclonal rabbit anti-GFAP; 1:1000; DAKO, Carpinteria, CA). Myelination was estimated by detection of myelin oligodendrocyte glycoprotein (MOG; 1:20, monoclonal mouse anti-MOG immunoglobulin $\left.\mathrm{G}[\mathrm{IgG}]^{38}\right)$. The oligodendrocyte progenitor response was evaluated by immunostaining for neural antigen 2 (NG2; polyclonal rabbit anti-NG2 ectodomain; 1:500; gift from William Stallcup, La Jolla, CA). Accumulation of phosphorylated tau was examined using antibodies against

FIG. 1. Characterization of the progression of pathology in the corpus callosum in a repetitive mild traumatic brain injury (r-mTBI) model. (A and B) Post-surgical data for r-mTBI. Apnea was not detected (nd) in r-sham mice (A), but was significantly increased after the first and the second impact in r-mTBI mice (A). The righting time is significantly delayed in r-mTBI versus r-sham mice (B). (C-E) Immunohistochemistry for $\beta$-APP is present mainly in neuronal cell bodies in $\mathrm{r}$-sham mice $(\mathrm{C})$. $\beta$-APP accumulations are readily detected in damaged axons (arrows) in r-mTBI mice acutely (D), but less marked by 7 days post-injury (E). (F-H) Immunohistochemistry for CD11b identifies microglia, which exhibit a resting phenotype in $\mathrm{r}$-sham mice $(\mathrm{F})$ with progressive activation evident at $24 \mathrm{~h}(\mathrm{G})$ and 7 days $(\mathrm{H})$. (I-K) Immunohistochemistry for GFAP identifies astrocytes that have normal morphology in r-sham mice (I) and increasing cells with a reactive phenotype in r-mTBI mice between $24 \mathrm{~h}(\mathrm{~J})$ and 7 days $(\mathrm{K})$. (L-O) Quantification of immunohistochemistry in the corpus callosum. Axonal profiles with $\beta$-APP accumulations were increased very early after r-mTBI mice (L). Astrogliosis increases over the first week after r-mTBI (M). Microglial activation also increases significantly over the first week based on area of CD11b immunoreactivity $(\mathrm{N})$ and an earlier reaction is detected by counting activated cells, which have intense CD11b and shorter, thicker processes (O). Dashed lines outline the corpus callosum. Values are mean \pm standard error of the mean; $n=30$ for post-surgical data; $n=5$ per condition for histology data; scale bar $(\mathrm{E}),(\mathrm{H})$, and $(\mathrm{K})=50 \mu \mathrm{m} ; * p<0.05 ; * * p<0.01 ; * * * p<0.001$; $* * * * p<0.0001$. $\beta$-APP, beta amyloid precursor protein; GFAP, glial fibrillary acidic protein; $r$-sham, repetitive sham. 

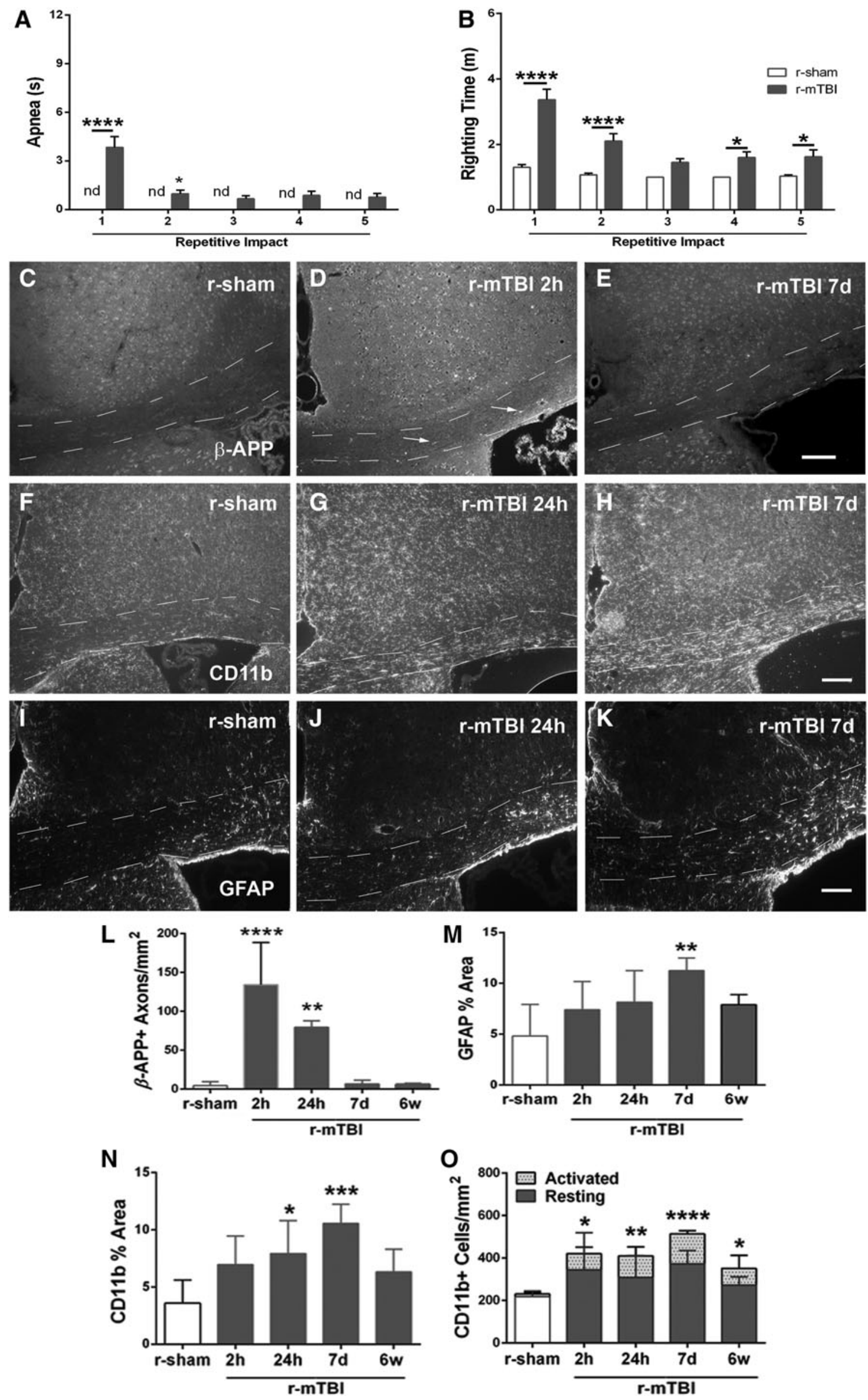
anti-phospho-Ser396Tau (mouse monoclonal IgG, 355300; 1:400; Thermo Fisher Scientific, Waltham, MA) and AT8 antibody (mouse monoclonal anti-phospho-PHF-tau pSer202+Thr205; 1:400; Thermo Fisher Scientific).

Astrogliosis, microglia/macrophage activation, and myelination were estimated from the area of immunoreactivity for GFAP, CD11b, or MOG within the CC or medial cortex using Metamorph (Molecular Devices, Downingtown, PA) as previously detailed. ${ }^{20,38}$ In addition, microglia/macrophage cells were also quantified by manual counting of the morphological phenotype for resting, activated, or amoeboid stages. ${ }^{20}$ Among mice perfused for postimaging analysis, $1 \mathrm{r}$-sham and $1 \mathrm{r}$-mTBI mouse were omitted because of technical concerns limiting quantification across the full set of regions in the double immunostain for CD11b and GFAP at the coronal levels matched to DTI-DKI slice at bregma. Damaged axons were quantified based on $\beta$-APP immunoreactivity, which was counted as a single damaged axon for accumulation as a large end bulb or as multiple swellings aligned along a longitudinal axonal profile.

Thy1-YFP-16 mice were used to visualize a high density of neurons in their entirety, including axons, cell bodies, and dendritic processes. Sections were prepared as above for immunohistochemistry. Nuclei were stained blue with 4',6-diamidino-2phenylindole (DAPI; Sigma-Aldrich) whereas rough endoplasmic reticulum in the cytoplasm of the cell bodies was stained red with a fluorescent version of Nissl histological stain (NeuroTrace red; Thermo Fisher Scientific). Damaged axons were quantified based on YFP in axons with swellings using the same morphological characteristics as for $\beta$-APP above.

Bright-field images were captured using an Olympus Nanozoomer (Olympus, Center Valley, PA). Fluorescent wide-field images were acquired on an Olympus IX-70 microscope (Olympus). Optical image fluorescent image stacks were acquired using a Zeiss LSM 700 (Carl Zeiss Microscopy, Thornwood, NY).

\section{Social interaction assessment}

The social interaction assessment was carried out using a threechamber sociability test apparatus (Stoelting Co., Wood Dale, IL) as previously described, with minor modification. ${ }^{39,40}$ The test mouse was first placed in the middle chamber and allowed $10 \mathrm{~min}$ to explore all chambers for habituation to the apparatus. A small wire enclosure was introduced to each of the side chambers and the mouse was allowed to explore all chambers for $10 \mathrm{~min}$ for habituation to the enclosures as novel objects. Social approach was then tested by placing an unfamiliar male C57BL/6J mouse (stranger) into one of the wire enclosures. The test mouse was again placed into the middle chamber and allowed to explore among the three chambers for $10 \mathrm{~min}$. The movement of the test mouse was video recorded to determine the time related to social approach (defined as movement toward, circling, or sniffing of the stranger mouse in the wire enclosure) using ANY-Maze software (Stoelting Co.) as analyzed by an observer blinded to the treatment condition. Mice were run in two independent cohorts of 12 mice that each had 6 mice assigned to each condition. The total assay included 24 mice, with 12 injured and 12 matched sham mice.

\section{Complex wheel-running assay}

Motor skill learning and bilateral sensorimotor coordination were assessed using a complex wheel-running assay. ${ }^{41-43}$ Running wheels were placed in home cages equipped with an optical sensor to detect wheel revolutions and determine the daily maximal running velocity using Activity Monitoring Software (Lafayette Instruments, Lafayette, IN) over a 3-week period. A regular "training" wheel with evenly spaced rungs was in the cages for 1 week to reduce variability in running behavior. Mice then underwent $r$-mTBI or $r$-sham procedures. Training wheels were then replaced with "complex" wheels, which have irregularly spaced rungs. ${ }^{41,42}$ Mice were run in two independent cohorts that each had 6 mice assigned to each condition. The total assay included 24 mice, with 12 injured and 12 matched sham mice. Social interaction cohorts were separate mice from those used on the wheel assay.

\section{Statistical analysis}

All data analyses were performed with GraphPad Prism software (version 6; GraphPad Software, Inc., La Jolla, CA). Two-way repeated measures analysis of variance (ANOVA) with post-hoc Bonferroni comparison was used to compare TBI with yoked shams across time points for longitudinal analyses. One-way ANOVA was used to compare data among multiple groups for a single condition or time point of tissue analysis, which included three tissue sections/mouse. Post-hoc comparisons were made among the relevant pairs (each TBI model vs. matched sham; single vs. repetitive TBI models). The Student's $t$-test was used to compare between two groups for a single condition or time point. Pearson's correlation was used for analysis of the relationship between radiological and pathological findings. Statistical significance was defined as $p<0.05$. MRI quantitative analysis was performed independent of post-imaging tissue analysis. All quantification was performed with blinding for the condition.

\section{Results}

\section{Initial characterization of repetitive mild traumatic brain injury severity based on progression of pathology in the corpus callosum}

The post-surgical data demonstrate apnea (Fig. 1A) and a delayed righting reflex (Fig. 1B) after r-mTBI compared to the r-sham procedures, which indicates concussive effects across this series of impacts. Mice were prepared for tissue analysis at multiple time points after r-mTBI to characterize the injury severity based on the extent of axon damage, astrogliosis, and microglial activation in the corpus callosum at the coronal level of impact at bregma (Fig. 1C-O). Accumulations of $\beta$-APP demonstrated axon damage with impaired fast axonal transport at early times after r-mTBI (Fig. 1C-E,L). By $2 \mathrm{~h}$ after the fifth impact in $\mathrm{r}$-mTBI mice, $\beta$-APPlabeled axons were significantly increased in the corpus callosum (Fig. 1L). Also, astrogliosis (Fig. 1I-K) and microglial activation (Fig. 1F-H) increased in the corpus callosum of r-mTBI mice by 7 days post-injury, as demonstrated by increases in the GFAP-immunolabeled area (Fig. 1M), CD11b-immunolabeled area (Fig. 1N), and density of activated microglia (Fig. 1O). Astrogliosis and microglial activation were less marked at 6 weeks post-injury (Fig. 1M,N) whereas the density of activated microglia continued to be significantly increased from r-sham levels (Fig. 1O). Prussian blue reaction, which detects hemosiderin-laden macrophages as evidence of previous bleeding, indicated a lack of microhemorrhages whereas the nuclear counterstain demonstrated normal adult tissue cytoarchitecture (Fig. 2).

\section{Diffusion imaging detects mainly cortical abnormalities after repetitive mild traumatic brain injury}

Longitudinal MRI analysis for both DTI and DKI techniques was tested as a method to identify microstructural changes in the corpus callosum and medial cerebral cortex induced by r-mTBI (Fig. 3). Mainly cortical changes were observed after r-mTBI. The only DTI or DKI parameter with a significant change in the corpus callosum on post-hoc analysis was a decrease in FA at week 6 (Fig. 3D). In contrast, cortical changes in both DTI and DKI were observed after r-mTBI (Fig. 3L-V). FA was reduced in the cortex at 

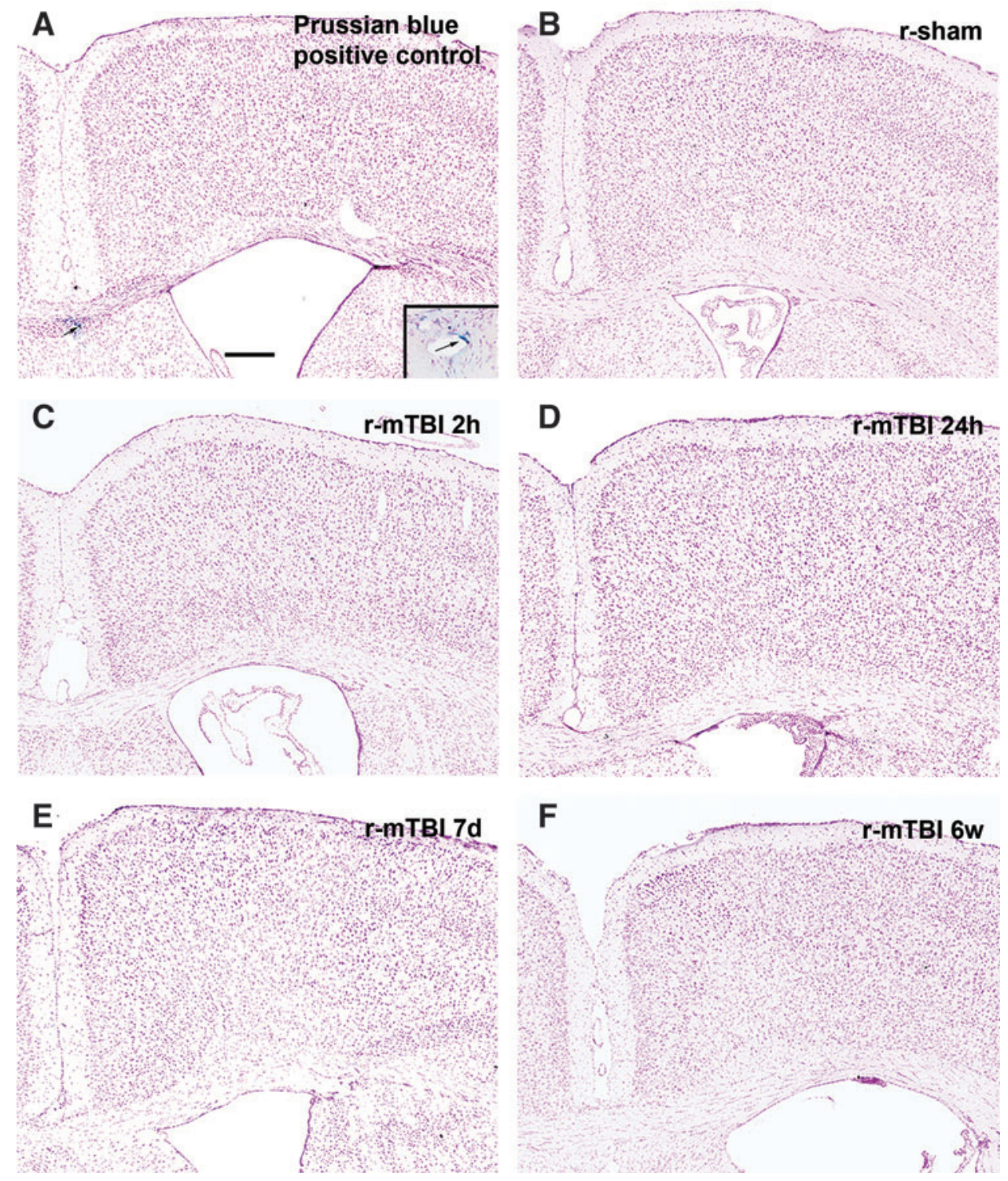

FIG. 2. Microhemorrhages are not observed after r-mTBI. Prussian blue was used to detect hemosiderin-laden macrophages that are indicative of previous hemorrhage or bleeding. A positive control for the Prussian blue reaction (arrowed and inset) is shown from a mouse with an injury that was more severe than those used in this study (A). No positive staining was present in either the corpus callosum or cortex in r-sham mice $(\mathbf{B})$ or after $\mathrm{r}$-mTBI at any time points examined $(\mathbf{C}-\mathbf{F}) . n=3$; scale bar $=250 \mu \mathrm{m}$. d, days; r-mTBI, repetitive mild traumatic brain injury; r-sham, repetitive sham; w, weeks.

FIG. 3. Diffusion imaging abnormalities after r-mTBI. Corpus callosum (CC) analysis is shown in upper graphs (A-K), for CC region outlined in yellow on upper image) and medial cerebral cortex values are shown in lower graphs ( $\mathbf{L}-\mathbf{V}$, for cortical region outlined in yellow on the lower image). Each region is shown at the coronal level containing the anterior commissure, which is under the impact site at bregma. r-sham and r-mTBI mice were scanned as yoked pairs with all mice being scanned at four time points-baseline (BL) and post-injury at 3 days, 6 days, and 6 weeks. Five coronal slices (one rostral to bregma, one at bregma $=0$, and 3 caudal to bregma) were analyzed. (A-D) FA values in the $\mathrm{CC}$ showing all five coronal slices with locations noted as relative to bregma. A significant reduction in FA was noted in r-mTBI mice only at the bregma level at 6 weeks (D). (E-K) CC values in the individual coronal slice at bregma are shown across time points for the full set of parameters analyzed-diffusion tensor imaging (FA, MD, AD, and RD) and diffusion kurtosis imaging $\left(\mathrm{MK}, \mathrm{K}_{\mathrm{a}}\right.$, and $\mathrm{K}_{\mathrm{r}}$ ). No parameters show a significant injury effect that reaches significance at any of the post-injury time points. (L-O) Analysis across coronal slices in the medial cortex shows a significant reduction in FA in r-mTBI mice versus sham near the impact site on days 3 and $6(\mathrm{M}$ and N). (P-S) Analysis of the individual slice under the impact site across time points for all diffusion parameters shows a significant injury effect in the axial dimension for both $\mathrm{AD}$ with diffusion tensor imaging $(\mathrm{R})$ and $\mathrm{K}_{\mathrm{a}}$ with diffusion kurtosis imaging (U). Values are mean \pm standard deviation, $n=6$ for each condition at each time point; ${ }^{*} p<0.05 ; * *<<0.01$. $\mathrm{AD}$, axial diffusivity; FA, fractional anisotropy; $\mathrm{K}_{\mathrm{a}}$, axial kurtosis; $\mathrm{K}_{\mathrm{r}}$, radial kurtosis; $\mathrm{MD}$, mean diffusivity; MK, mean kurtosis; RD, radial diffusivity; $r$-mTBI, repetitive mild traumatic brain injury; $r$-sham, repetitive sham. 
days 3 and 6 in coronal slices near the impact site in r-mTBI mice (Fig. 3M,N). However, these FA changes are relatively small, and statistical significance could potentially be affected by variation in the yoked sham cohort during the longitudinal study. Analysis of the DTI and DKI subparameters further refined the cortical changes after r-mTBI. DTI showed significantly decreased AD values at all time points post-TBI (Fig. 3R), with no changes in RD (Supplementary Fig. 3; see online supplementary material at http://www .liebertpub.com). DKI also showed a significant complementary change in the axial dimension after r-mTBI based on increased $\mathrm{K}_{\mathrm{a}}$ values at day 6 (Fig. 3U). Taken together, these DTI and DKI findings confirm relatively little corpus callosum damage.

Repetitive mTBI (r-mTBI): Corpus Callosum (CC) white bars $=r$-sham; colored bars $=r-m T B I$
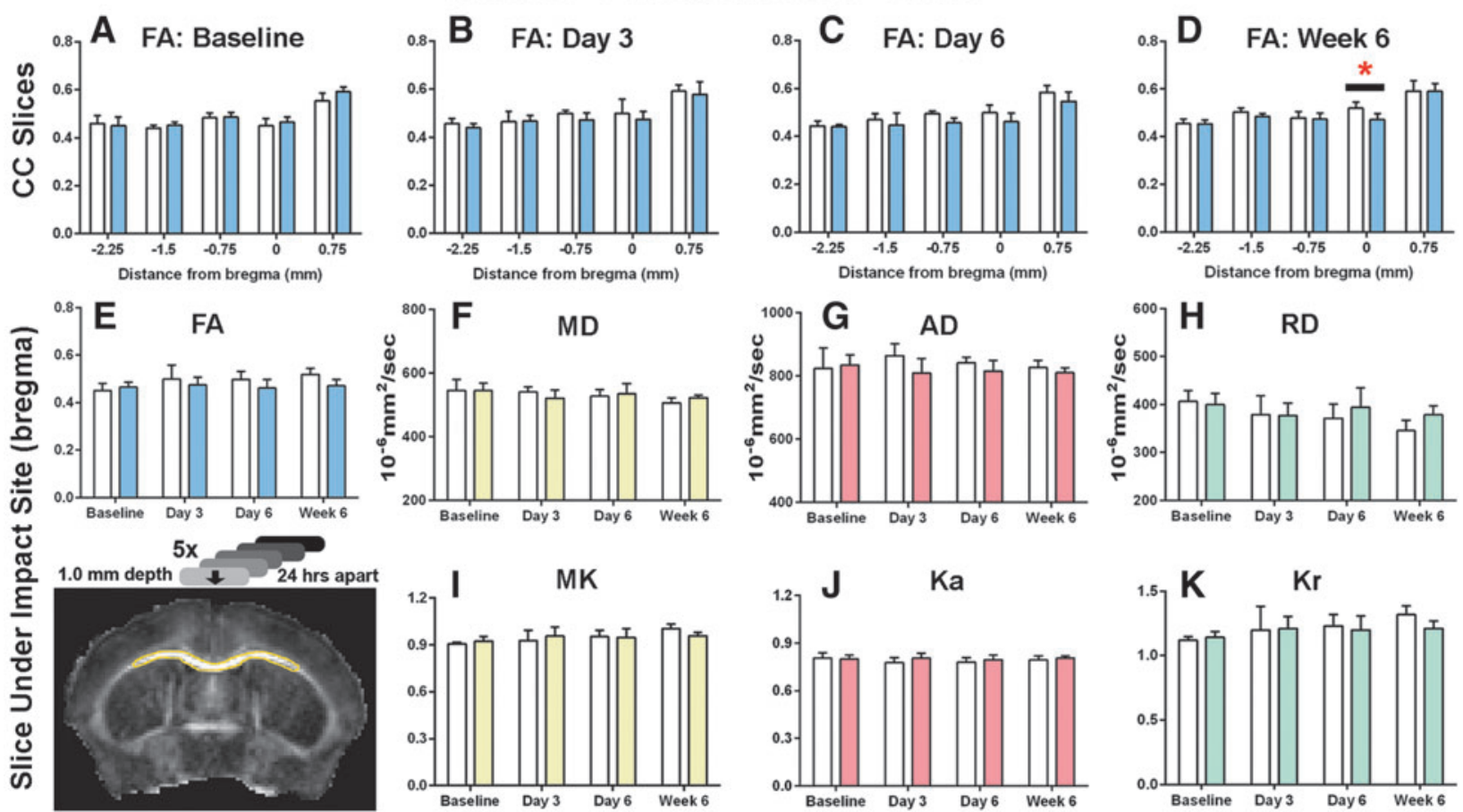

Repetitive mTBI (r-mTBI): Cerebral Cortex white bars $=r$-sham; colored bars $=r-m T B I$
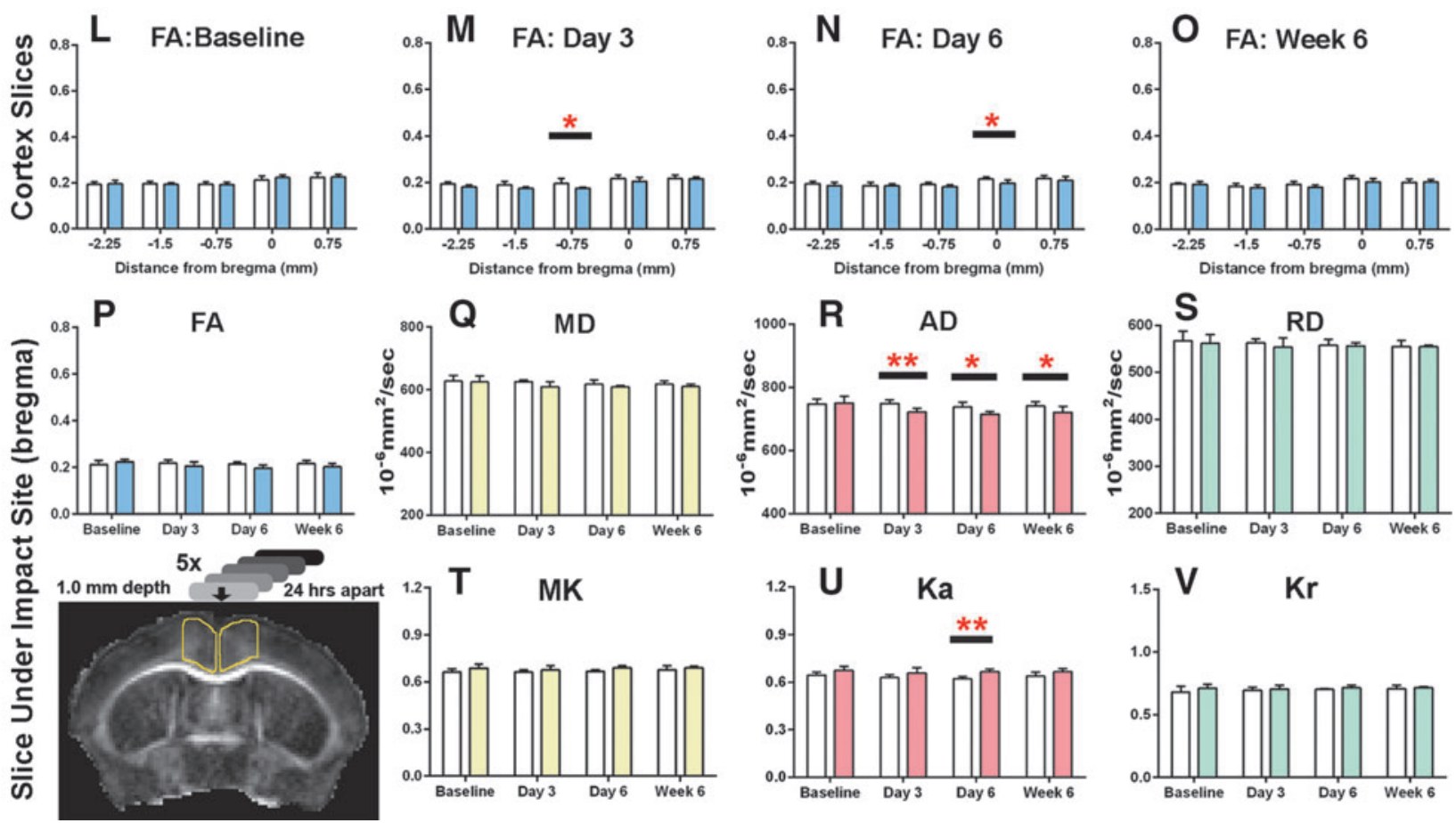
Conversely, and unexpectedly, our diffusion analysis revealed cortical abnormalities in this r-mTBI model.

To test the specificity of these findings in r-mTBI, we performed several additional analyses. Further r-mTBI analysis of diffusion DEC maps (Fig. 4A,B) demonstrated high anisotropy in the corpus callosum as well as marked directionality in the medial region of the cerebral cortex, which supports effective DTI and DKI analyses in these cortical areas. Structural analysis after r-mTBI using $\mathrm{T}_{2^{-}}$ weighted images and calculated $\mathrm{T}_{2}$ maps did not detect any overt structural changes or evidence of edema or microhemorrhages in the cortex under the impact site for any time point or condition (Fig. 4C,D and data not shown). The overlying scalp shows $\mathrm{T}_{2}$ hyperintensity attributed to the impacts onto the skin in the r-mTBI mice, which are not observed in r-sham mice (Fig. 4C,D).

\section{Post-imaging analysis of repetitive mild traumatic brain injury pathology in corpus callosum and cortex}

After completing the final MRI scan at the 6-week time point, mice were perfused for post-imaging analysis of the underlying pathology in the corpus callosum and cortical ROIs from the DTI and DKI analysis. We first examined neuroinflammation using CD11b and GFAP for detection of microglia and astrocytes, respectively. The r-mTBI tissues from our longitudinal MRI analysis showed mild, but persistent, neuroinflammation in the corpus
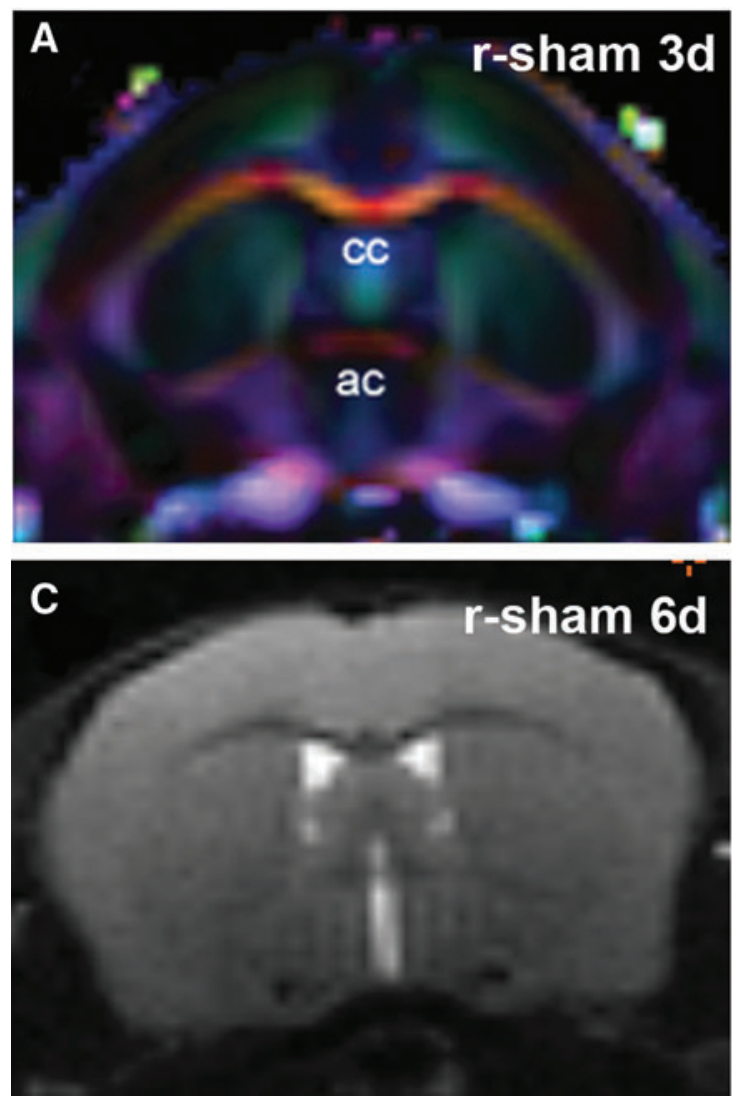

callosum (Fig. 5A,B). Specifically, in r-mTBI mice compared to r-sham mice, the percent area immunolabeled was increased for CD11b (Fig. 5C). Morphological classification of individual CD11b-labeled cells also showed that the number of activated microglia increased after r-mTBI (Fig. 5D). Amoeboid CD11blabeled cells were not found in any condition or time point (data not shown). After r-mTBI, astrogliosis in the corpus callosum was not significantly different from the r-sham condition (Fig. 5E). Overall, in the r-mTBI mice, neuroinflammation in the corpus callosum was much less robust relative to the s-mTBI model, which was used as a positive control comparison for corpus callosum pathology detectable by DTI (Supplementary Figs. 2 and 3) (see online supplementary material at http://www.liebert pub.com). Neuroinflammation in medial cortical regions, that is, directly under the impact site, in r-mTBI mice, showed a significant increase in microglial activation, but not astrogliosis (Fig. 5F-H). In contrast, the single-injury model did not exhibit significant neuroinflammation in the cortex with matched analysis of immunoreactivity for either CD11b (Supplementary Fig. 3F,G) (see online supplementary material at http://www .liebertpub.com) or GFAP (Supplementary Fig. 3H) (see online supplementary material at http://www.liebertpub.com) and did not have cortical abnormalities for DTI or DKI analysis (Supplementary Fig. 2) (see online supplementary material at http:// www.liebertpub.com).
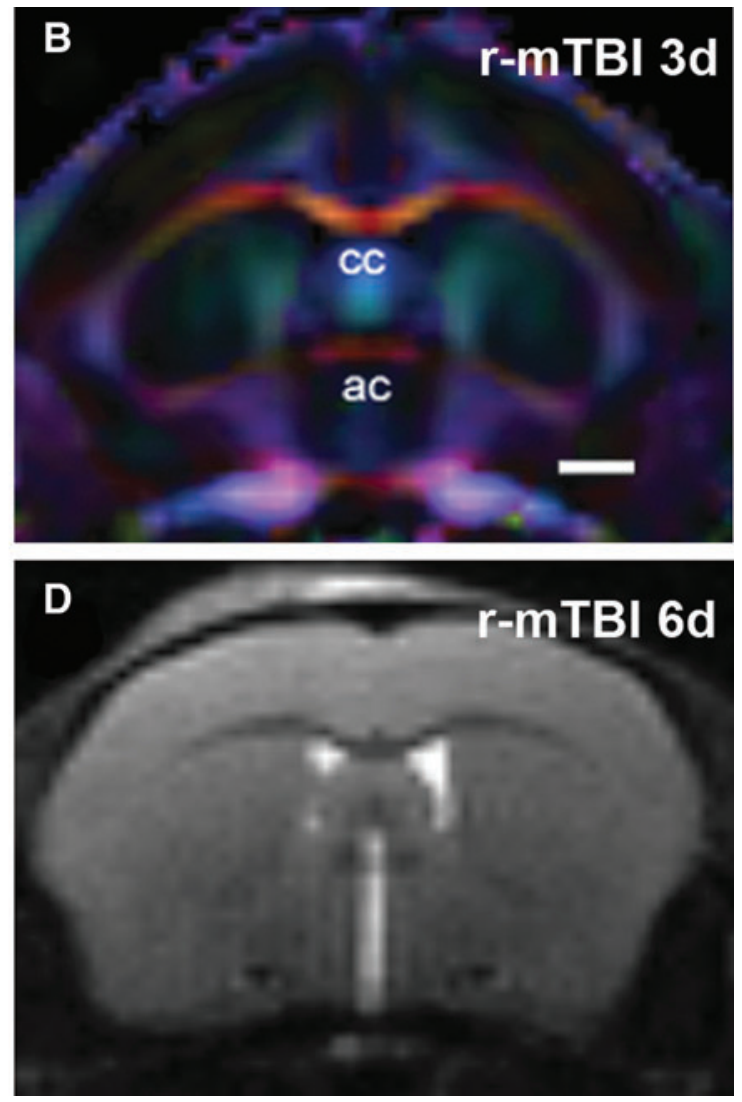

FIG. 4. In vivo diffusion direction encoded color (DEC) maps and T2-weighted imaging of r-mTBI. (A and B) DEC maps show the coronal level that includes the anterior commissure (ac), which aligns with bregma at the site of impact, 3 days after r-sham injury (A) or $r$ mTBI (B). The corpus callosum (cc) shows a high level of anisotropy. Note that the degree of anisotropy in the medial cortex is greater than in more lateral cortical regions. (C and $\mathbf{D})$ Representative T2-weighted images at the coronal level of impact do not show signs of edema or overt structural damage in either the corpus callosum or cortex after r-sham injury (C) or r-mTBI (D). Images show 6 days post-TBI when significant FA changes are found in the medial cortex after r-mTBI. Scale bar $=1 \mathrm{~mm}$. Colors indicate fiber direction: red: medial-lateral; blue: anterior-posterior; and green: superior-inferior. d, days; r-mTBI, repetitive mild traumatic brain injury; r-sham, repetitive sham. 

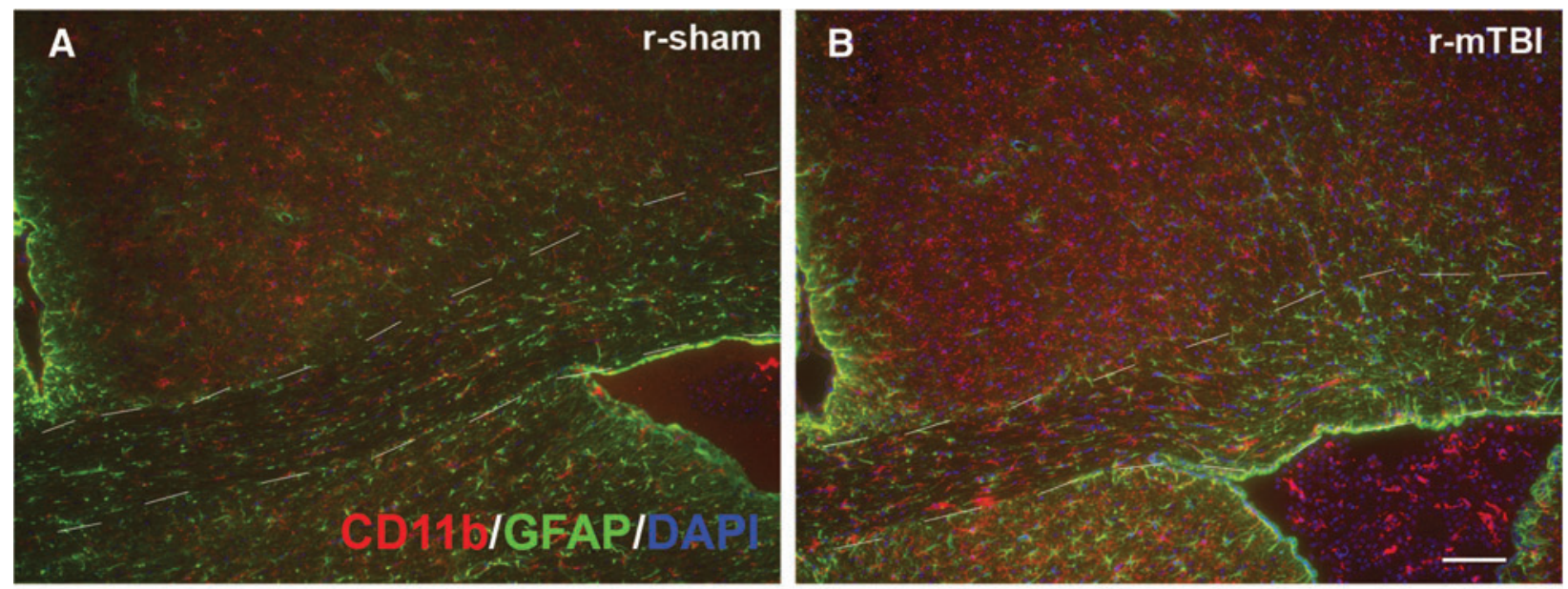

Corpus Callsoum
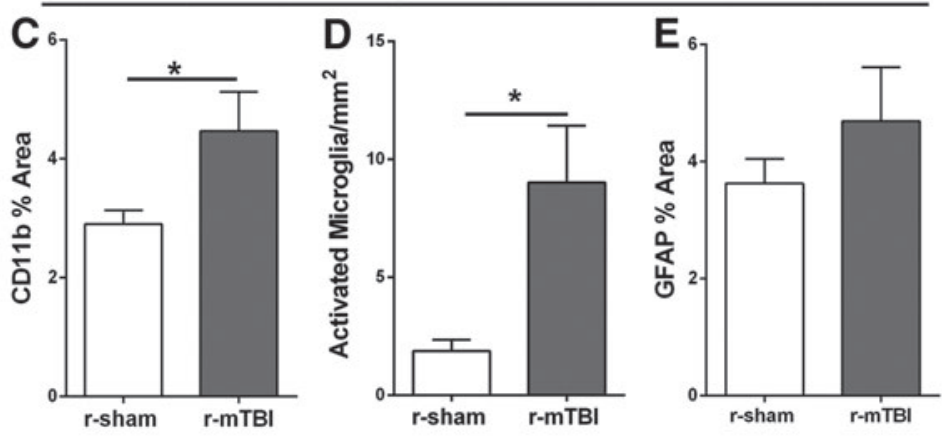

Cerebral Cortex
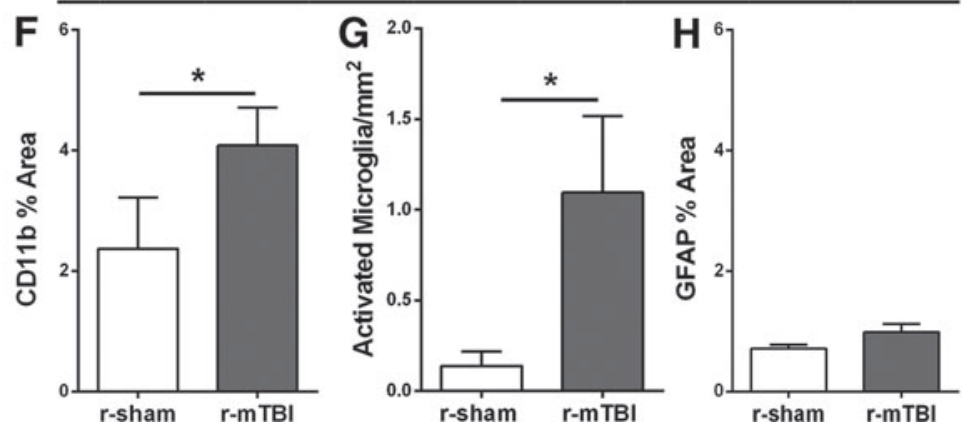

FIG. 5. Post-imaging neuroinflammation in the corpus callosum and cortex after r-mTBI. Immunohistochemistry was used to detect microglia/macrophage cells with CD11b and astrocytes with GFAP in mice perfused after the final imaging scan, that is, 6 weeks postinjury or sham procedure. (A and B) Slight astrogliosis and microglia/macrophage activation were evident in r-mTBI mice (B) compared to r-sham mice (A). (C-H) Quantitative analysis shows a significant increase in microglial activation in the corpus callosum of r-mTBI mice relative to sham $(\mathrm{C}$ and $\mathrm{D})$. Astrogliosis in the corpus callosum was not significantly increased after $\mathrm{r}-\mathrm{mTBI}(\mathrm{E})$. In the cortex, the r-mTBI mice had a significant increase in the area of CD11b immunolabeling and in the number of activated microglia (F and $\mathrm{G})$. Astrogliosis in the medial cortex was not significantly increased after r-mTBI $(\mathrm{H})$. Dashed lines outline the corpus callosum. Values are mean \pm standard error of the mean; $n=5$; scale bar $=50 \mu \mathrm{m} ;{ }^{*} p<0.05$. DAPI, 4',6-diamidino-2-phenylindole; GFAP, glial fibrillary acidic protein; r-mTBI, repetitive mild traumatic brain injury; r-sham, repetitive sham.

We next examined changes related to myelination (Fig. 6). Immunohistochemistry for MOG myelin protein correlates well with electron microscopy and Luxol fast blue myelin stain to estimate demyelination within the corpus callosum. ${ }^{44,45}$ After r-mTBI, MOG immunolabeling was examined both in the corpus callosum as well as in the cortex to explore the basis for the changes observed in cortical DTI/DKI (Fig. 6A,B). MOG in r-mTBI mice was not changed from r-sham levels for the corpus callosum (Fig. 6E), and the reduction in the cortex did not reach significance (Fig. 6G; $p=0.083$; power $=99 \%$ ). Post-imaging analysis of the s-mTBI model cohort for MOG immunostaining detected subtle diffuse demyelination (Supplementary Fig. 4) (see online supplementary material at http://www.liebertpub.com), consistent with demyelination observed by electron microscopy in this model at 6 weeks post-TBI. ${ }^{31}$

NG2 cells give rise to new oligodendrocytes for remyelination and are also an important reactive cell population after cortical injury. ${ }^{46-48}$ Our previous studies in the s-mTBI model found that NG2 cell density increased in the corpus callosum and cortex 

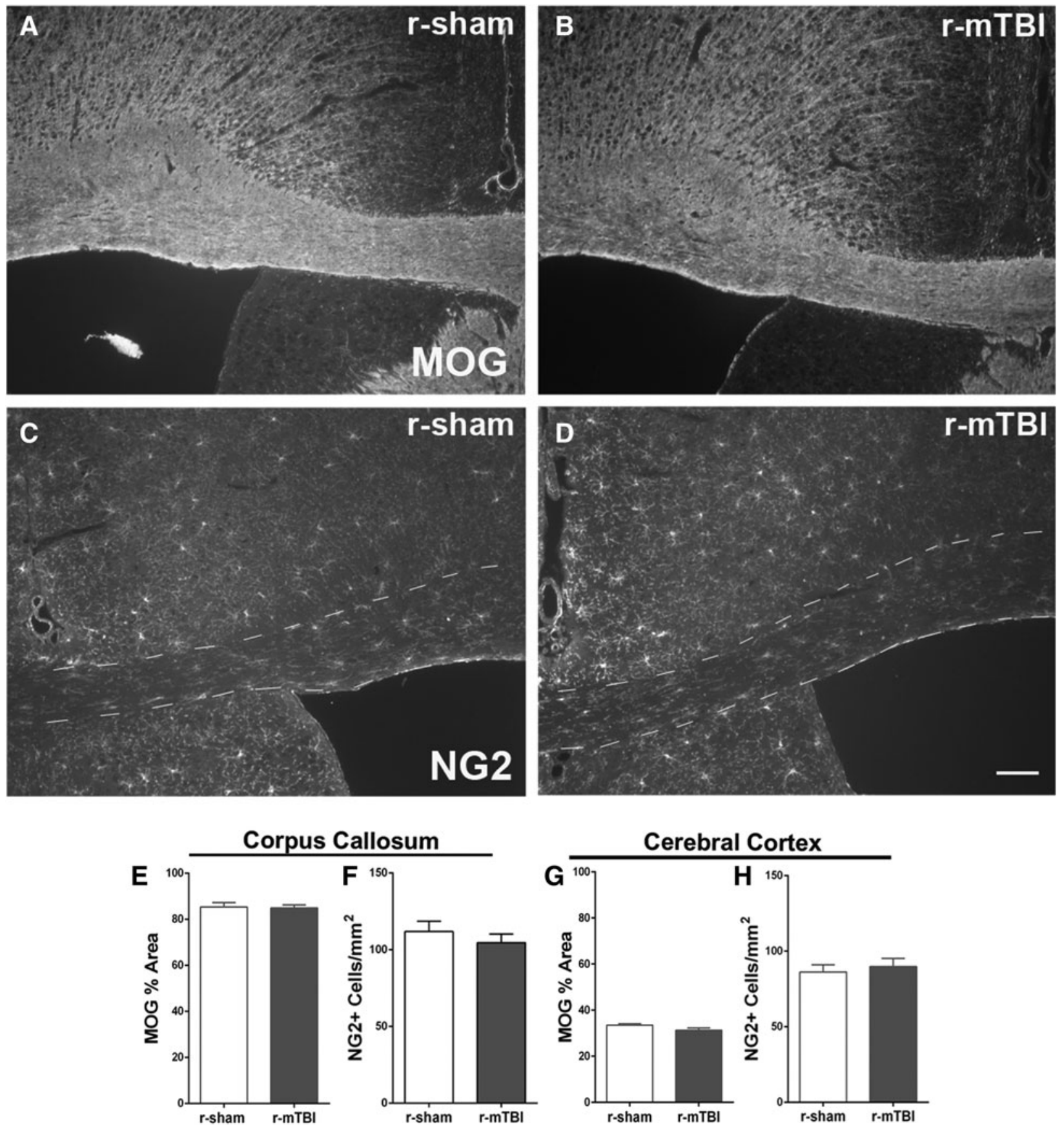

FIG. 6. r-mTBI does not alter myelination at 6 weeks post-injury. (A and B) Immunohistochemistry for myelin oligodendrocyte glycoprotein (MOG) to study myelination status post-imaging. No change of myelination was visible in either corpus callosum or cortex after r-mTBI (B) compared to r-sham (A), which was confirmed by quantification of MOG (E and $\mathbf{G})$. (C and D) Oligodendrocyte progenitors, detected by immunolabeling for NG2 (C and D), also were not changed in either corpus callosum $(\mathbf{F})$ or cortex $(\mathbf{H})$ after rmTBI compared to r-sham. Dashed lines outline the corpus callosum. $n=6$ for MOG; $n=3$ for NG2; values are mean \pm standard error of the mean; scale bars $=50 \mu \mathrm{m}$. NG2, neural antigen 2; r-mTBI, repetitive mild traumatic brain injury; r-sham, repetitive sham.

during the initial weeks post-injury with less of an elevation by 6 weeks post-injury. ${ }^{20,49}$ In r-mTBI mice, the NG2 cell population was not affected in the corpus callosum or cortex in the postimaging 6-week cohort (Fig. 6C,D,F,H).

Further analysis of tau pathology was performed in the postimaging 6-week cohort as a potential feature that may produce cortical changes after repetitive injury (Fig. 7). However, immunoreactivity for phosphorylated tau epitopes was not detected in r-mTBI mice using a pSer396 antibody (Fig. 7A) or AT8 antibody (data not shown). The sham condition also did not exhibit immunoreactivity for phosphorylated tau (data not shown). P301S human tau mice were used as a positive control using the same immunohistochemical protocols and showed clear tau pathology in cortical neurons for both pSer396 (Fig. 7B) and AT8 (data not shown).

Correlation studies were performed to more directly interpret the cortical abnormalities identified in the DTI-DKI analysis 

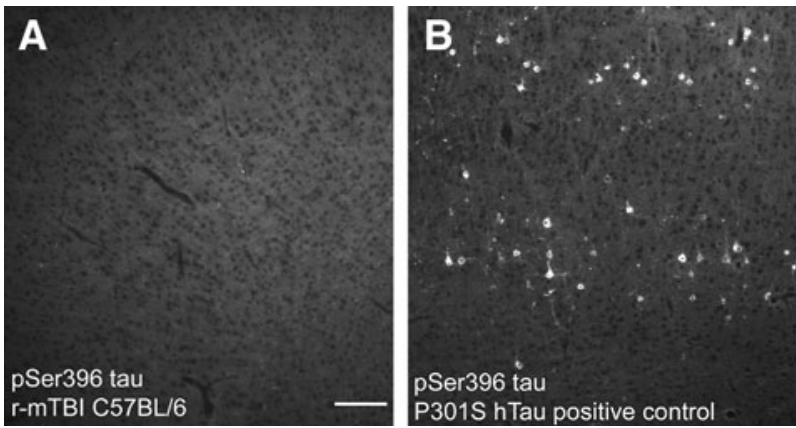

FIG. 7. Tau pathology is not observed after r-mTBI. Immunohistochemistry for phosphorylated serine 396 tau shows a lack of immunolabeling in the cortex of mice perfused postimaging at 6 weeks after r-mTBI (A). In contrast, the positive control P301S human tau tissues show high levels of phosphorylated serine 396 tau in cortical neurons $(\mathbf{B})$. Scale bar $=100 \mu \mathrm{m}$. r-mTBI, repetitive mild traumatic brain injury.

relative to the post-imaging pathological findings (Table 1; Fig. 8). At this 6-week time point, the significant changes between r-mTBI and r-sham were decreased AD values (Fig. 3R) and increased microglial activation (Fig. 5F). Values for these two parameters were strongly correlated (Table 1 ), and CD11b variation contributed to over $70 \%$ of the variation in AD based on the $r^{2}$ analysis (Fig. 8D). In contrast, cortical AD values did not correlate well with values for GFAP or MOG immunoreactivity (Table 1; Fig. 8E,F).

Next, we further examined the cortical changes after r-mTBI using Thy1-YFP-16 mice (Fig. 9), which have a high density of neurons with yellow fluorescent labeling. The labeling in cortical neurons enables visualization of the dendritic arbor and axonal processes, including callosal axons. ${ }^{50}$ Nissl staining in r-mTBI mice at 3 days post-injury and in matched r-sham mice showed a low density of neuronal cell bodies in layer I and a much higher density in layers II/II, indicating generally normal neuronal cytoarchitecture in the superficial cortex under the site of impact

Table 1. Radiological-Pathological Correlation For the Cortical Region at 6-Week Time Point

\begin{tabular}{|c|c|c|c|c|c|c|}
\hline & \multicolumn{2}{|c|}{$C D 11 b \%$ area } & \multicolumn{2}{|c|}{ GFAP \% area } & \multicolumn{2}{|c|}{$M O G \%$ area } \\
\hline & r-sham & $r-m T B I$ & r-sham & $r-m T B I$ & r-sham & $r-m T B I$ \\
\hline FA & 0.251 & -0.752 & $<0.001$ & -0.074 & 0.257 & 0.759 \\
\hline MD & -0.409 & -0.788 & 0.267 & 0.086 & -0.381 & 0.599 \\
\hline $\mathrm{AD}$ & -0.019 & -0.853 & 0.120 & 0.011 & -0.230 & -0.195 \\
\hline RD & -0.548 & -0.487 & 0.306 & 0.298 & -0.471 & 0.755 \\
\hline MK & 0.209 & 0.655 & -0.135 & 0.224 & 0.107 & -0.515 \\
\hline $\mathrm{K}_{\mathrm{a}}$ & -0.319 & 0.649 & 0.351 & 0.111 & -0.280 & -0.533 \\
\hline $\mathrm{K}_{\mathrm{r}}$ & 0.161 & -0.256 & -0.105 & -0.018 & -0.015 & 0.437 \\
\hline
\end{tabular}

DTI-DKI parameters were analyzed relative to post-imaging neuropathology quantification. Coefficient of correlation $(r)$ values demonstrate the strength of linear relationship. A strong correlation is found for the relationship between $\mathrm{AD}$ and $\mathrm{CD} 11 \mathrm{~b}$ (bold), which were the only two parameters with a statistically significant difference between $\mathrm{r}$-sham and r-mTBI at 6 weeks post-injury.

FA, fractional anisotropy; MD, mean diffusivity; AD, axial diffusivity; $\mathrm{RD}$, radial diffusivity; MK, mean kurtosis; $\mathrm{K}_{\mathrm{a}}$, axial kurtosis; $\mathrm{K}_{\mathrm{r}}$, radial kurtosis; r-sham, repetitive sham; r-mTBI, repetitive mild traumatic brain injury; GFAP, glial fibrillary acidic protein; MOG, myelin oligodendrocyte glycoprotein; DTI, diffusion tensor imaging; DKI, diffusion kurtosis imaging.
(Fig. 9A,B). In deeper cortical regions, the Thy1-YFP labeling was very dense without overt disruption of cytoarchitecture in r-mTBI, which appeared similar to r-sham (Fig. 9A,B). In r-sham mice, normal YFP distribution in axons is evident in higher-magnification images of the corpus callosum (Fig. 9C) and the medial cortex (Fig. 9E). In cortical regions of r-mTBI mice, YFP accumulation in some labeled axons was observed as axonal swellings (Fig. 9B,F). YFP expression revealed conspicuous axonal swellings in the corpus callosum of r-mTBI mice (Fig. 9D). Quantitative analysis of Thy1-YFP mice in the medial cortical region analyzed by MRI, which was comprised of cingulate cortex and M2 motor cortex, showed a significant increase of damaged YFP-labeled axonal segments in r-mTBI mice compared to the r-sham condition (Fig. 9G). As a point of validation, a parallel cohort of Thy1-YFP mice was examined with the s-mTBI model (Supplementary Fig. 5A,B) (see online supplementary material at http://www .liebertpub.com), in which axon damage in the corpus callosum has been previously characterized by electron microscopy. ${ }^{31}$ The s-mTBI mice, in which more-extensive corpus callosum pathology was expected, indeed exhibited more marked axon damage in the corpus callosum (Supplementary Fig. 5A,B) (see online supplementary material at http://www.liebertpub.com), compared to the r-mTBI mice (Fig. 9D). However, in the medial cortex, levels of damaged axonal segments labeled with YFP were similar in r-mTBI (Fig. 9G) and s-mTBI (Supplementary Fig. 5C) (see online supplementary material at http://www.liebertpub.com) models. Therefore, axonal damage in the cortex may contribute to DTI effects, but does not fully explain the reduction in cortical AD, which was only detected in this r-mTBI model.

\section{Social interaction deficit resulting from repetitive mild traumatic brain injury}

Finally, behavioral assessments were targeted to the MRI ROIs to detect potential functional deficits in r-mTBI mice. The corpus callosum overlying the lateral ventricles is associated with motor skill learning that can be assessed using a running wheel with irregularly spaced rungs. ${ }^{43}$ Over a 3 -week period, the r-mTBI mice appeared similar to $r$-sham mice in mastering the complex runningwheel task (Fig. 10A,B). The medial cortical region under the impact site used in this r-mTBI model includes the anterior cingulate cortex, which is considered a hub of social behaviors. ${ }^{51} \mathrm{~A}$ three-chamber social apparatus was used to compare the time each mouse spent interacting with an empty carrier or with a stranger mouse inside the carrier (Fig. 10C). The r-sham mice showed the normal preference to spend time interacting with the stranger mouse (Fig. 10C). The interaction time decreased from 190.3 \pm $13.8 \mathrm{sec}$ in $\mathrm{r}$-sham mice to $127.9 \pm 13.5 \mathrm{sec}$ in $\mathrm{r}$-mTBI mice (Fig. 10C), indicating impaired social approach behavior after $r-$ mTBI. A cohort of mice subjected to the s-mTBI model that produces more-extensive damage in the corpus callosum than the cortex (Supplementary Figs. 2 and 3) (see online supplementary material at http://www.liebertpub.com) did not result in behavioral changes in either wheel running or social interaction (Supplementary Fig. 6) (see online supplementary material at http://www .liebertpub.com) and serves as a negative control comparison. Overall, the r-mTBI impairment on social behavior supports the interpretation that r-mTBI induces significant cortical changes.

\section{Discussion}

Our findings demonstrate the utility of longitudinal DTI combined with DKI for noninvasive analysis of white matter 

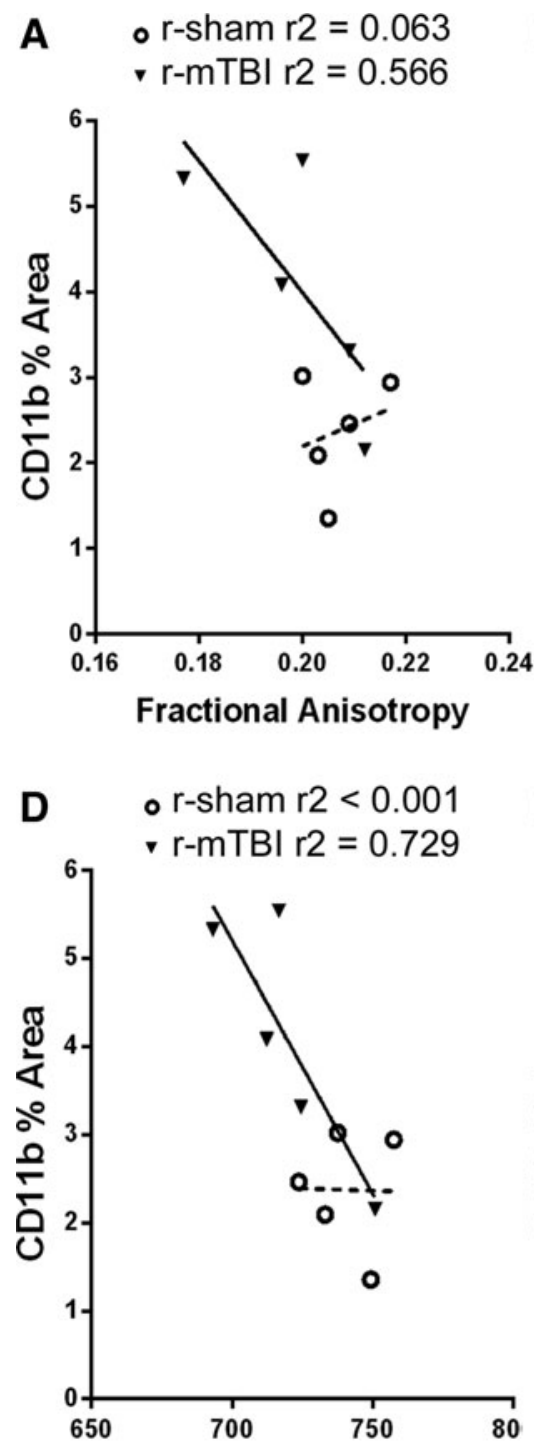

Axial Diffusivity $\left(10^{-6} \mathrm{~mm}^{2} / \mathrm{sec}\right.$
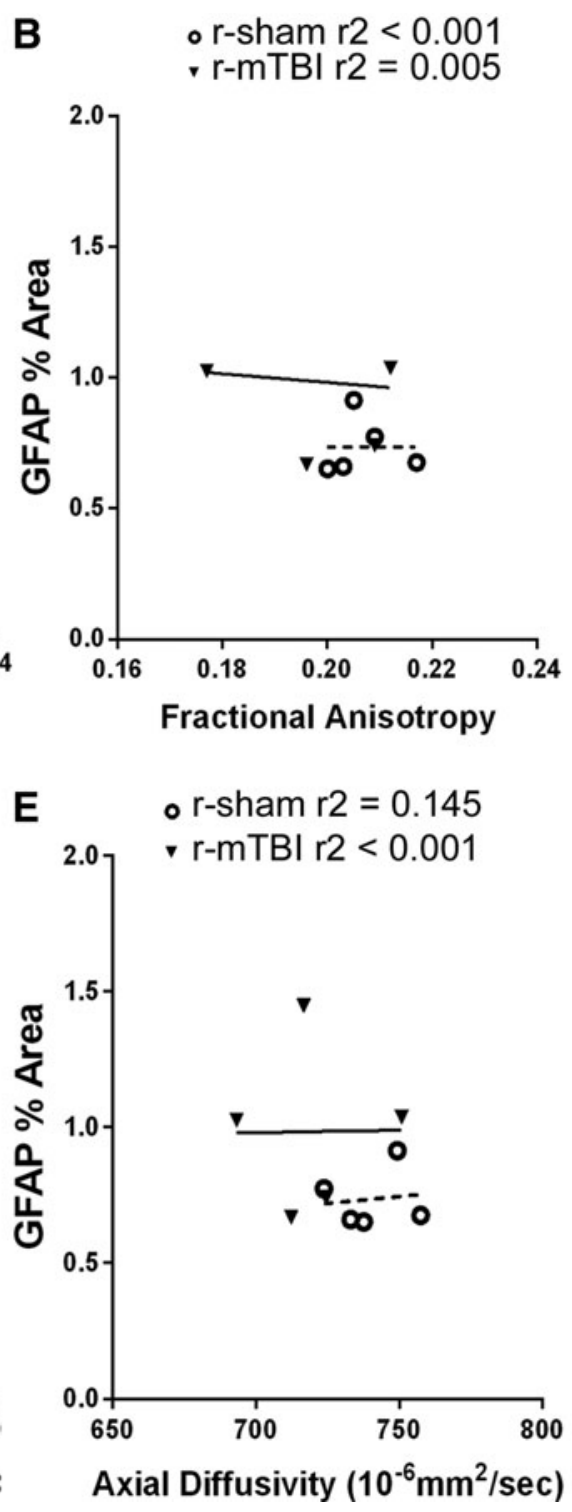
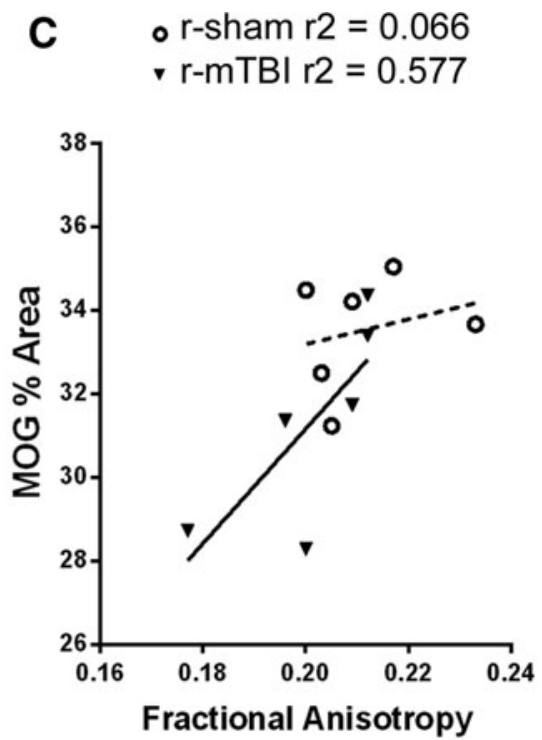

$\mathbf{F}$

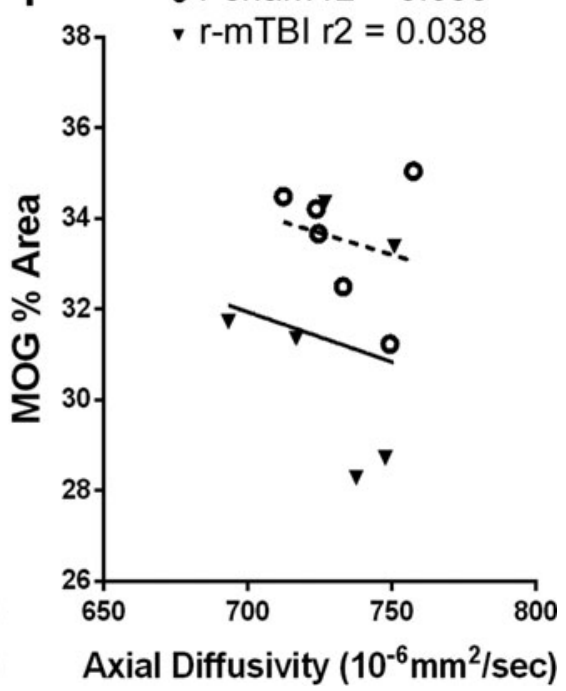

FIG. 8. Neuroimaging-neuropathological correlations for medial cortex effects after r-mTBI. To complement the correlation data shown in Table 1, individual values for each mouse are graphed for FA (A-C) and AD (D-F), given that the relationships most relevant for interpretation of the significant changes from r-mTBI, resulting in reduced cortical AD (Fig. 3) and increased CD11b immunoreactivity (Fig. 5). High CD11b values correlate strongly with reduced FA values (A) and reduced AD values (D). Coefficient of determination values $\left(r^{2}\right)$ show that approximately $72 \%$ of the variation in AD can be accounted for by the relationship with CD11b values. GFAP immunoreactivity did not show specific correlation with FA (B) or AD (E). MOG myelin values increased with increasing FA (C), but were not correlated with $\mathrm{AD}(\mathrm{F})$. AD, axial diffusivity; FA, fractional anisotropy; GFAP, glial fibrillary acidic protein; MOG, myelin oligodendrocyte glycoprotein; r-mTBI, repetitive mild traumatic brain injury; r-sham, repetitive sham.

injury as well as more-subtle cortical abnormalities resulting from repetitive concussive impacts. These neuroimaging changes were supported by post-imaging pathological analysis of the corpus callosum and cerebral cortex at 6 weeks post-injury. This r-mTBI model had minimal DTI/DKI changes in the corpus callosum. Yet, the r-mTBI mice showed significant changes in the cortex for axial parameters with both DTI (AD) and DKI $\left(\mathrm{K}_{\mathrm{a}}\right)$. Cortical changes in AD after r-mTBI correlated strongly with a significant microglial reaction on post-imaging histopathology. These cortical differences were supported by corresponding deficits in social behavior, which involves cortical regions under the impact site.
As a supplemental analysis, using a previously characterized single-impact model, ${ }^{31}$ we performed neuroimaging out to a subacute time point of 6 weeks with both DTI and DKI analysis followed by post-imaging tissue analysis. This single-impact model served as a positive control for corpus callosum pathology (Supplementary Fig. 3) (see online supplementary material at http:// www.liebertpub.com) detectable by DTI (Supplementary Fig. 2) (see online supplementary material at http://www.liebertpub.com), to more accurately interpret the mainly negative findings for DTI and DKI in the corpus callosum of r-mTBI mice (Fig. 3).

Our radiological findings in the s-mTBI model are relatively consistent with recent studies of single-impact injuries, although 

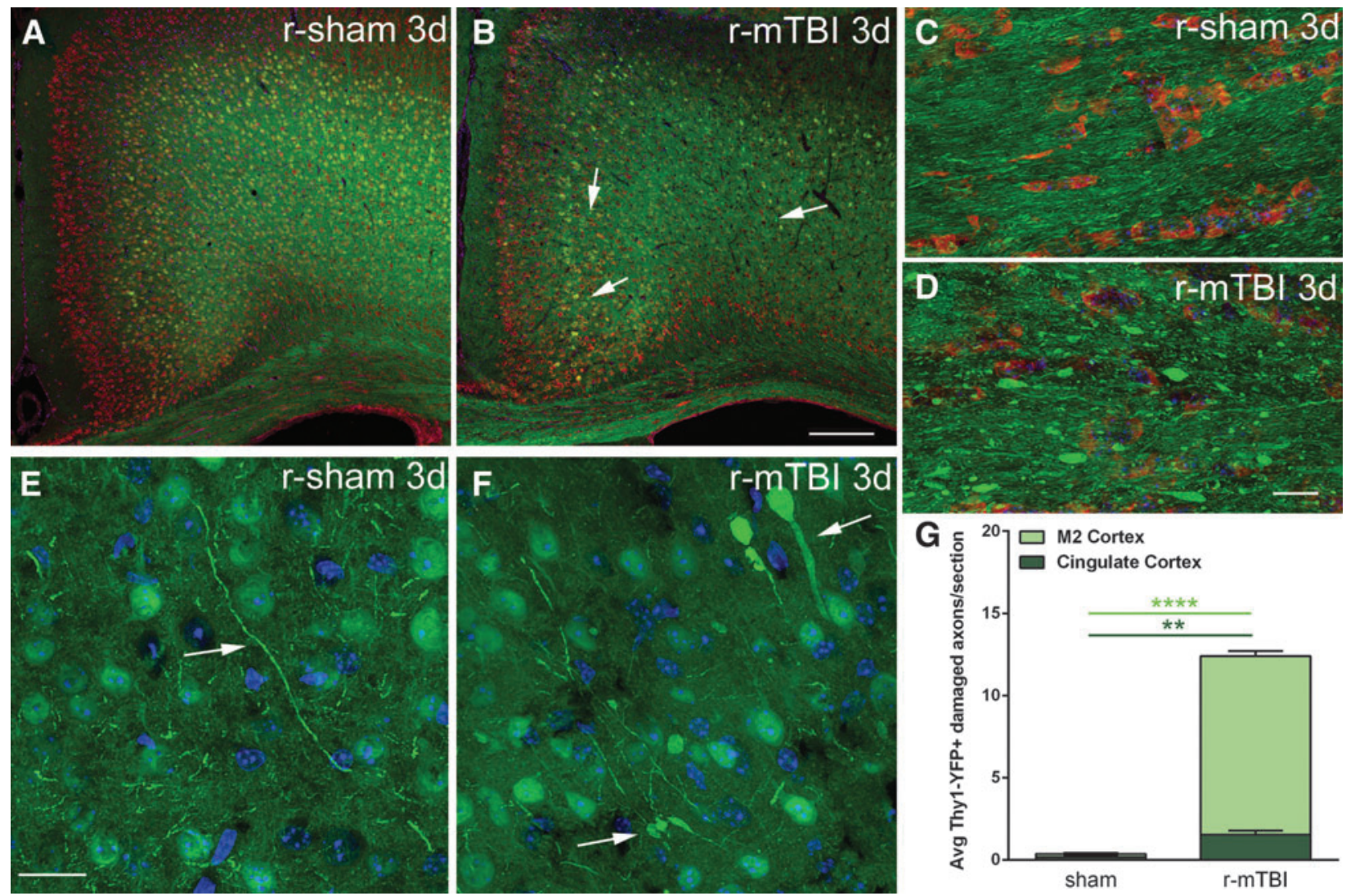

FIG. 9. Thy1-YFP-16 mice show axonal pathology in the cortex and corpus callosum after r-mTBI. (A and B) Thy1-YFP (green) shows labeling of neurons throughout the cortex along with axons projecting through the corpus callosum in mice perfused 3 days after r-sham (A) or r-mTBI (B) procedure. Nissl labeling (red) of the cytoplasmic rough endoplasmic reticulum along with DAPI nuclei counterstaining (blue) shows the overall cortical cytoarchitecture, which is not markedly disrupted after r-mTBI. Arrows (B) point to examples of swollen segments of damaged axons. (C and D) At higher magnification, Thy1-YFP (green) labeled axons in the corpus callosum exhibit a generally uniform longitudinal profile in r-sham mice (C) in contrast to the numerous swollen axonal segments observed in r-mTBI mice (D). (E and F) High-magnification Thy1-YFP (green) cortical regions analyzed in r-sham mice (E) and rmTBI mice (F). Large swellings and thickened Thy1-YFP axonal profiles are evident in the cortex of r-mTBI mice (F, arrows) compared to the thin processes of Thy1-YFP axons in r-sham mice (E, arrow). Lack of DAPI nuclear labeling (blue) shows that enlarged Thy1YFP regions (F) are not cell bodies. (G) Quantification of damaged axonal profiles (swollen, axonal bulbs, and varicosities) labeled with Thy1-YFP in the cingulate and M2 motor cortices that comprise the region of interest in the medial cortex for DTI/DKI analyses. A significant increase of damaged axons was observed in $\mathrm{r}-\mathrm{mTBI}$ mice compared to $\mathrm{r}$-sham. Values are mean \pm standard error of the mean; $n=3$; three to nine sections per animal. **p<0.01; ****p $<0.0001$. Scale bars $=200 \mu \mathrm{m}$ (A and B) and $20 \mu \mathrm{m}(\mathrm{C}-\mathrm{F}$ ). Avg, average; d, days; DAPI, 4',6-diamidino-2-phenylindole; DKI, diffusion kurtosis imaging; DTI, diffusion tensor imaging; r-mTBI, repetitive mild traumatic brain injury; r-sham, repetitive sham.

differences in pathology must be taken into consideration. Closed head impact in rats resulted in reduced FA in the corpus callosum, which corresponded with axonal injury. ${ }^{52} \mathrm{CCI}$ in rats produces a cortical contusion lesion along with pathology in a widespread distribution of white matter tracts and corresponds with bidirectional changes in FA. ${ }^{14}$ In this report, FA was reduced in the corpus callosum, but was increased in subcortical white matter as well as in some white matter tracts. Therefore, reduced FA was predicted to reflect fiber disorganization versus reorganization and/or higher fiber density in tracts with increased FA. ${ }^{14}$

The novel r-mTBI model was initially developed in the current study based on analysis of the rostral corpus callosum (Fig. 1) and then expanded to overlying cortical regions to explore potential pathology after repetitive impacts. The 1-day interval choice was supported from our recent findings that glucose uptake is decreased at $24 \mathrm{~h}$ post-TBI ${ }^{30}$ and a second injury at $24 \mathrm{~h}$ worsens outcome. ${ }^{53}$
This r-mTBI model is anterior, but otherwise similar, to several other more posterior repetitive mTBI models, ${ }^{54-57}$ suggesting that paradigms of at least three to five mild impacts with 1- to 3-day intervals are effective and practical. Our expectation was that the impact used in the r-mTBI model would result in relatively mild initial damage in the corpus callosum whereas repetition may promote progression toward chronic white matter pathology, including neuroinflammation.

Indeed, the r-mTBI model showed no overt structural damage based on Prussian blue (Fig. 2) and Nissl staining (Fig. 9) or T2 MRI (Fig. 4). $\beta$-APP analysis at $24 \mathrm{~h}$ showed that the r-mTBI (Fig. 1L) resulted in less than half the amount of initial axon damage in the corpus callosum than was observed after s-mTBI in our previous study. ${ }^{20}$ A progression toward chronic white matter pathology was indicated by a significant reduction of FA that was only observed in the corpus callosum at 6 weeks post-injury in 

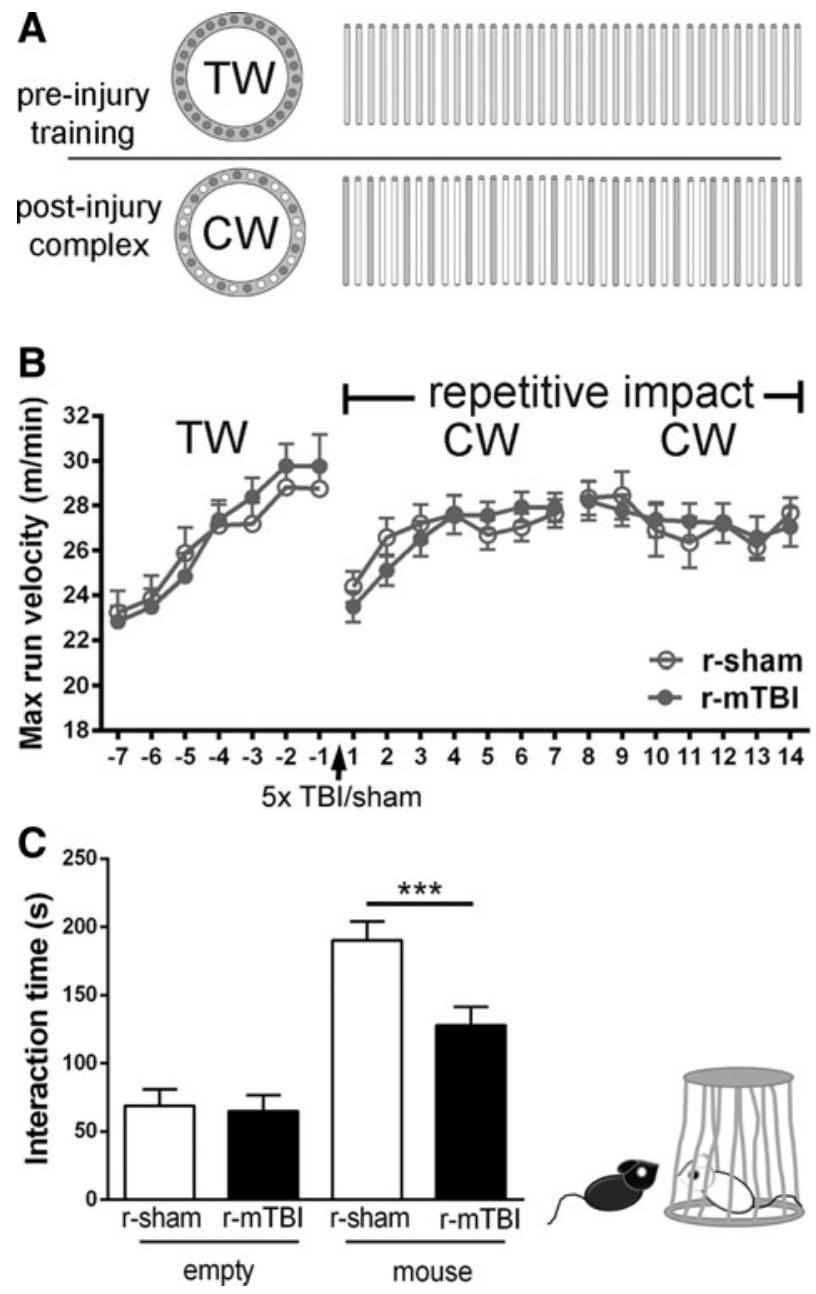

FIG. 10. r-mTBI results in social deficits. Mice were assessed for motor and social behavior related to functions of cortical regions under the impact site at bregma and corresponding areas of the corpus callosum. (A and $\mathbf{B}$ ) The motor task used a running wheel sequence as shown by the pattern of wheel rungs (A) across a 3-week time line. Mice were first provided training wheels (TW; regular rung pattern) to stabilize baseline running behavior for 1 week preceding $\mathrm{r}$-sham/r-mTBI (days -7 to 0 ). After $\mathrm{r}$-sham $/ \mathrm{r}$ mTBI, mice were provided complex wheels (CW; nonuniform rung spacing) for 1 week (days $0-7$ ). Mice must learn to adapt to the complex rung pattern to master this motor skill. The second week (days 8-14) on the complex wheels assesses the velocity plateau as a measure of bilateral sensorimotor coordination. The cohorts ( $n=12$ per condition) did not show differences in any phase on the training or complex wheel after r-mTBI (B). (C) A three-chamber sociability test was used to test social behavior at 3 weeks after r-mTBI. The r-sham mice spent the majority of time interacting with the carrier containing a stranger mouse rather than the empty carrier. The r-mTBI mice showed a significant reduction in social approach behaviors. The r-mTBI mice spent less time interacting with the unfamiliar mouse, as compared to the behavior of r-sham. Values are mean \pm standard error of the mean; $n=12 ; * * * p<0.001$. Max, maximum; r-mTBI, repetitive mild traumatic brain injury; r-sham, repetitive sham.

r-mTBI mice. However, at the 6-week time point, r-mTBI only slightly increased neuroinflammation in the corpus callosum (Fig. 5C,D), whereas the greatest changes in microglial activation and astrogliosis were observed at 1 week post-injury (Fig. 1M-O). Thus, DTI was unable to detect early changes in r-mTBI white matter pathology that appeared at chronic time points. Previous studies have commented on the limitations in the sensitivity of DTI, which is influenced by multiple pathological features, including axonal damage, changes in cellularity, and extracellular reorganization. ${ }^{9,14,16,58}$

The r-mTBI model produced significant radiological (Fig. 3) and pathological differences (Fig. 5) in cerebral cortex regions overlying the corpus callosum at coronal levels near the impact site. Further, r-mTBI injury resulted in social behavior deficits (Fig. 10) associated with neural circuits of this medial cortical region, which includes anterior cingulate cortex. The r-mTBI mice had slight, but significant, differences in cortical FA values that were driven by reduced $\mathrm{AD}$ (Fig. 3). DKI detects changes in the non-Gaussian component of diffusion at higher b-values and is expected to reflect changes in diffusional heterogeneity, which may correspond to microstructural complexity. ${ }^{26,59}$ DKI showed a corresponding change in $\mathrm{K}_{\mathrm{a}}$, which is consistent with effects in the axial direction (Fig. 3). These cortical findings are somewhat unusual in that gray matter generally has low anisotropy so that DTI analysis is not expected to be informative. However, some cortical regions have more structural directionality, such as the medial cortical region analyzed in this study (Fig. 4).

Although the r-mTBI changes in $\mathrm{AD}$ and $\mathrm{K}_{\mathrm{a}}$ may be relatively small, they may be particularly informative in cortical regions with sufficient anisotropy. In white matter, FA appears most informative because of the combination of pathological factors that can decrease axial values, including axon damage, and increase radial values, including demyelination. ${ }^{60,61}$ After r-mTBI, in cortical regions, demyelination was not observed (Fig. 6) and axon damage was similar to s-mTBI (Figs. 9 and Supplementary Fig. 5) (see online supplementary material at http://www.liebertpub .com)

Yet, cortical regions showed significantly reduced AD and increased $\mathrm{K}_{\mathrm{a}}$, which typically corresponds to axon damage. Neuroinflammation has also been shown to influence DTI and DKI parameters in TBI models. For example, several reports have correlated astrogliosis with increased FA, driven by increased $\mathrm{AD}$, or with kurtosis. ${ }^{18,26,52}$ In the current study, the decreased $\mathrm{AD}$ values in this medial cortical region correlated strongly with a significant increase in microglial reactivity on post-imaging tissue analysis (Table 1; Fig. 8A,D). It should be noted that diffusion studies have often evaluated FA values without reporting $\mathrm{AD}$ or $\mathrm{RD}$ changes, ${ }^{62}$ and this lack of specificity complicates the physiological interpretation of these diffusion changes. Even with clear changes in FA, AD, and RD throughout a time course of white matter disease, the specificity of each parameter is impaired when pathological features are present in combination. ${ }^{61}$ Previous animal and clinical studies have not necessarily agreed on $\mathrm{FA}, \mathrm{RD}$, or AD changes in gray matter postmTBI. ${ }^{27,63,64}$ Further studies are needed to more completely examine the full extent of cortical pathology across the post-injury time course after repetitive injury.

The pathological and radiological abnormalities observed in the anterior cingulate and motor cortices after r-mTBI could be a precursor to cortical thinning that has been associated with concussion. In collegiate football players with a history of concussion, MRI revealed significant cortical thinning in frontotemporal regions, including the rostral anterior cingulate cortex and motor cortex, without changes in white matter FA. ${ }^{65}$ The anterior cingulate region was also identified as a region of cortical atrophy in a 1-year longitudinal study of mTBI patients with a single episode of concussion. ${ }^{66}$ Cortical abnormalities after repetitive concussive 
injury have also been associated with hyperphosphorylated tau accumulations in neurons and astrocytes, particularly at the depths of sulci and around small blood vessels. ${ }^{67,68}$ In our analysis of rmTBI mice, hyperphosphorylated tau pathology was not evident using either AT8 staining or an antibody against phosphorylated serine 396 of tau (Fig. 7). Both antibodies readily detected tau pathology in transgenic P301S mice expressing mutant human tau, which served as a positive control. Therefore, tau pathology is unlikely to have contributed to the cortical abnormalities in AD and $\mathrm{K}_{\mathrm{a}}$ in our r-mTBI model. However, longer-term studies would be needed to fully appreciate the potential similarities of our r-mTBI findings to chronic-stage changes in TBI patients based on either cortical thinning or neurodegenerative disease.

To further understand the effects of the r-mTBI injury, functional assessments were targeted to the corpus callosum and cortical regions examined in our DTI/DKI analysis and post-imaging histopathology. The rostral area of corpus callosum is involved in motor skill learning on the complex running wheels. ${ }^{43}$ Axonal pathology in the corpus callosum or M2 motor cortex, which was evident at 3 days post-injury in Thy1-YFP-16 mice, did not impair motor skill learning (Fig. 10A,B). The r-mTBI mice had slower maximum velocities during the learning period of days 1-3 after introduction to the complex wheels, but the injury did not result in a statistical difference from sham. By day 4 on the complex wheels, the cohorts reached a plateau for the maximum velocity run each day, which was similar between injured and sham mice through 2 weeks post-injury. This plateau velocity has been shown to significantly decrease in conditions with extensive demyelination of the corpus callosum. ${ }^{41,42}$ The single-impact model with moreextensive corpus callosum pathology also did not alter the running on the complex wheel (Supplementary Fig. 6A) (see online supplementary material at http://www.liebertpub.com). The lack of impairment on both phases of this complex wheel assay indicates that either the current injuries do not impair function of a sufficient proportion of axons in each region or that broader pathology is needed to detect functional deficits with these assessment protocols.

The medial cortex region in our analysis includes the anterior cingulate cortex, which is involved in multiple cognitive domains and is considered a cortical hub for socially driven interactions in humans. ${ }^{51}$ In mice, deficits in social approach behavior are related to changes in intracellular signaling, cortical cytoarchitecture, and dendritic spine formation in the anterior cingulate cortex. ${ }^{69}$ The three-chambered task has been widely used to evaluate social interactions in mice. ${ }^{39,70,71}$ In TBI models, decreased social interaction has been reported in conditions of mild injury when combined with stress. ${ }^{40,72}$ We used this assessment of social approach behavior and found deficits after r-mTBI. Therefore, repetitive injury can impair social behavior without a stress paradigm. Social approach behavior was tested at 3 weeks post-injury or sham procedures as a time point that was midway between the 3-day and 6-week time points analyzed by MRI. This behavioral deficit at 3 weeks after r-mTBI indicates a potential predictive value of the 3- and 6-day abnormalities in DTI and DKI. These findings could be used to now develop an experimental design that would formally test predictive value in future studies.

Our findings highlight the need to evaluate changes in both white matter tracts and cortical regions post-mTBI. Using longitudinal MRI DTI combined with DKI, FA was most sensitive to varying severity of white matter injury (Fig. 3 and Supplementary Fig. 2) (see online supplementary material at http://www. liebertpub.com). Axial parameters, $\mathrm{AD}$ and $\mathrm{K}_{\mathrm{a}}$, were more informative for detecting subtle cortical abnormalities resulting from repetitive mild injury (Fig. 3). Cortical changes in AD after repetitive injury were strongly correlated with an indicator of a microglial reaction on post-imaging tissue analysis (Table 1; Fig. 8). The r-mTBI model used in the current study exhibits more pathology, based on $\beta$ APP analysis (Fig. 1), than a report of DTI changes in the cortex only in MD after repetitive mTBI. ${ }^{73}$ Our studies are consistent with recent work in fluorescent reporter mice that characterized the microglia reaction as sensitive to cortical damage in mTBI. ${ }^{74} \mathrm{~A}$ recent study of acute repetitive closed head TBI in mice also reported changes in RNA expression indicating activation of microglia in the cortex along with changes in ex vivo DTI AD. ${ }^{75}$ Therefore, repetitive concussive or subconcussive impacts may lead to a microglial reaction in the cortex that could be detected noninvasively with continued advances in the clinical application of diffusion imaging approaches. This cortical microglial reaction could have significant functional implications for behavioral deficits and cognitive impairment given the emerging roles of microglia in synaptic pruning and neurodegenerative disease. ${ }^{76-78}$

\section{Acknowledgments}

This study was funded by the Department of Defense within the Center for Neuroscience and Regenerative Medicine. We appreciate the assistance of Dr. Amanda Mierzwa, Laurel Beer, Tuan Le, and the Pre-clinical Core and Translational Imaging Core of the Center for Neuroscience and Regenerative Medicine. We thank Drs. R.P. Gullapalli and J. Zhuo (University of Maryland, Baltimore, MD) for providing the MATLAB code for DKI processing.

\section{Author Disclosure Statement}

No competing financial interests exist.

\section{References}

1. Clinical Tools for Provider. (2016). http://dvbic.dcoe.mil/ resources/clinical-tools (last accessed April 12, 2016).

2. DoD Worldwide Numbers for TBI. (2016). http://dvbic.dcoe.mil/ dod-worldwide-numbers-tbi (last accessed April 12, 2016).

3. Gardner, R.C., and Yaffe, K. (2015). Epidemiology of mild traumatic brain injury and neurodegenerative disease. Mol. Cell. Neurosci. 66, $75-80$.

4. McMahon, P., Hricik, A., Yue, J.K., Puccio, A.M., Inoue, T., Lingsma, H.F., Beers, S.R., Gordon, W.A., Valadka, A.B., Manley, G.T., and Okonkwo, D.O.; TRACK-TBI Investigators. (2014). Symptomatology and functional outcome in mild traumatic brain injury: results from the prospective TRACK-TBI study. J. Neurotrauma 31, 26-33.

5. Yuh, E.L., Cooper, S.R., Mukherjee, P., Yue, J.K., Lingsma, H.F., Gordon, W.A., Valadka, A.B., Okonkwo, D.O., Schnyer, D.M., Vassar, M.J., Maas, A.I., and Manley, G.T.; TRACK-TBI Investigators. (2014). Diffusion tensor imaging for outcome prediction in mild traumatic brain injury: a TRACK-TBI study. J. Neurotrauma 31, 14571477.

6. Ware, J.B., Biester, R.C., Whipple, E., Robinson, K.M., Ross, R.J., and Nucifora, P.G. (2016). Combat-related mild traumatic nrain injury: association between baseline diffusion-tensor imaging findings and long-term outcomes. Radiology 280, 212-219.

7. Kenney, K., Amyot, F., Haber, M., Pronger, A., Bogoslovsky, T., Moore, C., and Diaz-Arrastia, R. (2016). Cerebral vascular injury in iraumatic brain injury. Exp. Neurol. 275, 353-366.

8. Yang, S.T., Hsiao, I.T., Hsieh, C.J., Chiang, Y.H., Yen, T.C., Chiu, W.T., Lin, K.J., and Hu, C.J. (2015). Accumulation of amyloid in 
cognitive impairment after mild traumatic brain injury. J. Neurol. Sci. 349, 99-104.

9. Edlow, B.L., Copen, W.A., Izzy, S., Bakhadirov, K., van der Kouwe, A., Glenn, M.B., Greenberg, S.M., Greer, D.M., and Wu, O. (2016) Diffusion tensor imaging in acute-to-subacute traumatic brain injury: a longitudinal analysis. BMC Neurol. 16, 2.

10. Smits, M., Houston, G.C., Dippel, D.W., Wielopolski, P.A., Vernooij, M.W., Koudstaal, P.J., Hunink, M.G., and van der Lugt, A. (2011). Microstructural brain injury in post-concussion syndrome after minor head injury. Neuroradiology 53, 553-563.

11. Niogi, S.N., Mukherjee, P., Ghajar, J., Johnson, C., Kolster, R.A., Sarkar, R., Lee, H., Meeker, M., Zimmerman, R.D., Manley, G.T., and McCandliss, B.D. (2008). Extent of microstructural white matter injury in postconcussive syndrome correlates with impaired cognitive reaction time: a $3 \mathrm{~T}$ diffusion tensor imaging study of mild traumatic brain injury. AJNR. Am. J. Neuroradiol. 29, 967973 .

12. Adam, O., Mac Donald, C.L., Rivet, D., Ritter, J., May, T., Barefield M., Duckworth, J., LaBarge, D., Asher, D., Drinkwine, B., Woods, Y., Connor, M., and Brody, D.L. (2015). Clinical and imaging assessment of acute combat mild traumatic brain injury in Afghanistan. Neurology $85,219-227$.

13. Delano-Wood, L., Bangen, K.J., Sorg, S.F., Clark, A.L., Schiehser, D.M., Luc, N., Bondi, M.W., Werhane, M., Kim, R.T., and Bigler, E.D. (2015). Brainstem white matter integrity is related to loss of consciousness and postconcussive symptomatology in veterans with chronic mild to moderate traumatic brain injury. Brain Imaging Behav. 9, 500-512.

14. Harris, N.G., Verley, D.R., Gutman, B.A., and Sutton, R.L. (2016) Bi-directional changes in fractional anisotropy after experiment TBI: disorganization and reorganization? Neuroimage 133, 129_ 143.

15. Shenton, M.E., Hamoda, H.M., Schneiderman, J.S., Bouix, S., Pasternak, O., Rathi, Y., Vu, M.A., Purohit, M.P., Helmer, K., Koerte, I., Lin, A.P., Westin, C.F., Kikinis, R., Kubicki, M., Stern, R.A., and Zafonte, R. (2012). A review of magnetic resonance imaging and diffusion tensor imaging findings in mild traumatic brain injury. Brain Imaging Behav. 6, 137-192.

16. Eierud, C., Craddock, R.C., Fletcher, S., Aulakh, M., King-Casas, B., Kuehl, D., and LaConte, S.M. (2014). Neuroimaging after mild traumatic brain injury: review and meta-analysis. NeuroImage Clin. 4, 283-294.

17. Bazarian, J.J., Zhu, T., Blyth, B., Borrino, A., and Zhong, J. (2012) Subject-specific changes in brain white matter on diffusion tensor imaging after sports-related concussion. Magn. Reson. Med. 30, 171180 .

18. Budde, M.D., Janes, L., Gold, E., Turtzo, L.C., and Frank, J.A. (2011). The contribution of gliosis to diffusion tensor anisotropy and tractography following traumatic brain injury: validation in the rat using Fourier analysis of stained tissue sections. Brain 134, 2248 2260 .

19. Mayer, A.R., Ling, J., Mannell, M.V., Gasparovic, C., Phillips, J.P., Doezema, D., Reichard, R., and Yeo, R.A. (2010). A prospective diffusion tensor imaging study in mild traumatic brain injury. Neurology 74, 643-650.

20. Sullivan, G.M., Mierzwa, A.J., Kijpaisalratana, N., Tang, H., Wang, Y., Song, S.K., Selwyn, R., and Armstrong, R.C. (2013). Oligodendrocyte lineage and subventricular zone response to traumatic axonal injury in the corpus callosum. J. Neuropathol. Exp. Neurol. 72, 11061125 .

21. Hulkower, M.B., Poliak, D.B., Rosenbaum, S.B., Zimmerman, M.E., and Lipton, M.L. (2013). A decade of DTI in traumatic brain injury: 10 years and 100 articles later. AJNR. Am. J. Neuroradiol. 34, 2064 2074.

22. Glenn, G.R., Kuo, L.W., Chao, Y.P., Lee, C.Y., Helpern, J.A., and Jensen, J.H. (2016). Mapping the orientation of white matter fiber bundles: a comparative study of diffusion tensor imaging, diffusional kurtosis imaging, and diffusion spectrum imaging. AJNR. Am. J. Neuroradiol. 37, 1216-22.

23. Grossman, E.J., Jensen, J.H., Babb, J.S., Chen, Q., Tabesh, A., Fieremans, E., Xia, D., Inglese, M., and Grossman, R.I. (2013). Cognitive impairment in mild traumatic brain injury: a longitudinal diffusional kurtosis and perfusion imaging study. AJNR. Am. J. Neuroradiol. 34, 951-957.
24. Grossman, E.J., Ge, Y., Jensen, J.H., Babb, J.S., Miles, L., Reaume, J., Silver, J.M., Grossman, R.I., and Inglese, M. (2012). Thalamus and cognitive impairment in mild traumatic brain injury: a diffusional kurtosis imaging study. J. Neurotrauma 29, 2318-2327.

25. Stokum, J.A., Sours, C., Zhuo, J., Kane, R., Shanmuganathan, K., and Gullapalli, R.P. (2015). A longitudinal evaluation of diffusion kurtosis imaging in patients with mild traumatic brain injury. Brain Inj. 29, 47-57.

26. Zhuo, J., Xu, S., Proctor, J.L., Mullins, R.J., Simon, J.Z., Fiskum, G., and Gullapalli, R.P. (2012). Diffusion kurtosis as an in vivo imaging marker for reactive astrogliosis in traumatic brain injury. NeuroImage 59, 467-477.

27. Bouix, S., Pasternak, O., Rathi, Y., Pelavin, P.E., Zafonte, R., and Shenton, M.E. (2013). Increased gray matter diffusion anisotropy in patients with persistent post-concussive symptoms following mild traumatic brain injury. PLoS One 8, e66205.

28. Davenport, E.M., Apkarian, K., Whitlow, C.T., Urban, J.E., Jensen, J.H., Szuch, E., Espeland, M.A., Jung, Y., Rosenbaum, D.A., Gioia, G., Powers, A.K., Stitzel, J.D., and Maldjian, J.A. (2016). Abnormalities in diffusional kurtosis metrics related to head impact exposure in a season of high school varsity football. J. Neurotrauma 33, 2133 2146.

29. Allen, B., Ingram, E., Takao, M., Smith, M.J., Jakes, R., Virdee, K., Yoshida, H., Holzer, M., Craxton, M., Emson, P.C., Atzori, C., Migheli, A., Crowther, R.A., Ghetti, B., Spillantini, M.G., and Goedert, M. (2002). Abundant tau filaments and nonapoptotic neurodegeneration in transgenic mice expressing human P301S tau protein. J. Neurosci. 22, 9340-9351.

30. Selwyn, R., Hockenbury, N., Jaiswal, S., Mathur, S., Armstrong, R.C., and Byrnes, K.R. (2013). Mild traumatic brain injury results in depressed cerebral glucose uptake: an (18)FDG PET study. J. Neurotrauma 30, 1943-1953.

31. Mierzwa, A.J., Marion, C.M., Sullivan, G.M., McDaniel, D.P., and Armstrong, R.C. (2015). Components of myelin damage and repair in the progression of white matter pathology after mild traumatic brain injury. J. Neuropathol. Exp. Neurol. 74, 218-232.

32. Stejskal, E.O., and Tanner, J.E. (1965). Spin diffusion measurements: spin echoes in the presence of a time-dependent field gradient. J. Chem. Phys. 42, 288-292.

33. Hennig, J., Nauerth, A., and Friedburg, H. (1986). RARE imaging: a fast imaging method for clinical MR. Magn. Reson. Med. 3, 823833.

34. Pierpaoli, C., Walker, L., Irfanoglu, M.O., Barnett, A., Basser, P., Chang, L.-C., Koay, C., Pajevic, S., Rohde, G., Sarlls, J., and Wu, M. (2010). Tortoise: an integrated software package for processing of diffusion MRI data. In: ISMRM Annual Meeting: Stockholm, Sweden, p. 1597.

35. Pajevic, S., and Pierpaoli, C. (1999). Color schemes to represent the orientation of anisotropic tissues from diffusion tensor data: application to white matter fiber tract mapping in the human brain. Magn. Reson. Med. 42, 526-540.

36. Jensen, J.H., Helpern, J.A., Ramani, A., Lu, H., and Kaczynski, K (2005). Diffusional kurtosis imaging: the quantification of nongaussian water diffusion by means of magnetic resonance imaging. Magn. Reson. Med. 53, 1432-1440.

37. Stone, J.R., Singleton, R.H., and Povlishock, J.T. (2000). Antibodies to the C-terminus of the beta-amyloid precursor protein (APP): a site specific marker for the detection of traumatic axonal injury. Brain Res. $871,288-302$

38. Armstrong, R.C., Le, T.Q., Flint, N.C., Vana, A.C., and Zhou, Y.X. (2006). Endogenous cell repair of chronic demyelination. J. Neuropathol. Exp. Neurol. 65, 245-256.

39. Moy, S.S., Nadler, J.J., Perez, A., Barbaro, R.P., Johns, J.M., Magnuson, T.R., Piven, J., and Crawley, J.N. (2004). Sociability and preference for social novelty in five inbred strains: an approach to assess autistic-like behavior in mice. Genes Brain Behav. 3, 287302 .

40. Ojo, J.O., Greenberg, M.B., Leary, P., Mouzon, B., Bachmeier, C. Mullan, M., Diamond, D.M., and Crawford, F. (2014). Neurobehavioral, neuropathological and biochemical profiles in a novel mouse model of co-morbid post-traumatic stress disorder and mild traumatic brain injury. Front. Behav. Neurosci. 8, 213.

41. Hibbits, N., Pannu, R., Wu, T.J., and Armstrong, R.C. (2009). Cuprizone demyelination of the corpus callosum in mice correlates with 
altered social interaction and impaired bilateral sensorimotor coordination. ASN Neuro. 1, e00013.

42. Mierzwa, A.J., Zhou, Y.X., Hibbits, N., Vana, A.C., and Armstrong, R.C. (2013). FGF2 and FGFR1 signaling regulate functional recovery following cuprizone demyelination. Neurosci. Lett. 548, 280-285.

43. McKenzie, I.A., Ohayon, D., Li, H., de Faria, J.P., Emery, B., Tohyama, K., and Richardson, W.D. (2014). Motor skill learning requires active central myelination. Science 346, 318-322.

44. Armstrong, R.C., Le, T.Q., Frost, E.E., Borke, R.C., and Vana, A.C. (2002). Absence of fibroblast growth factor 2 promotes oligodendroglial repopulation of demyelinated white matter. J. Neurosci. 22, 8574-8585.

45. Lindner, M., Heine, S., Haastert, K., Garde, N., Fokuhl, J., Linsmeier, F., Grothe, C., Baumgartner, W., and Stangel, M. (2008). Sequential myelin protein expression during remyelination reveals fast and efficient repair after central nervous system demyelination. Neuropathol. Appl. Neurobiol. 34, 105-114.

46. Levine, J.M. (1994). Increased expression of the NG2 chondroitinsulfate proteoglycan after brain injury. J. Neurosci. 14, 4716-4730.

47. Hughes, E.G., Kang, S.H., Fukaya, M., and Bergles, D.E. (2013). Oligodendrocyte progenitors balance growth with self-repulsion to achieve homeostasis in the adult brain. Nat. Neurosci. 16, 668 676.

48. Susarla, B.T., Villapol, S., Yi, J.H., Geller, H.M., and Symes, A.J. (2014). Temporal patterns of cortical proliferation of glial cell populations after traumatic brain injury in mice. ASN Neuro. 6, 159-170.

49. Mierzwa, A.J., Sullivan, G.M., Beer, L.A., Ahn, S., and Armstrong, R.C. (2014). Comparison of cortical and white matter traumatic brain injury models reveals differential effects in the subventricular zone and divergent Sonic hedgehog signaling pathways in neuroblasts and oligodendrocyte progenitors. ASN Neuro. 6, 17590914 14551782.

50. Petraglia, A.L., Plog, B.A., Dayawansa, S., Dashnaw, M.L., Czerniecka, K., Walker, C.T., Chen, M., Hyrien, O., Iliff, J.J., Deane, R., Huang, J.H., and Nedergaard, M. (2014). The pathophysiology underlying repetitive mild traumatic brain injury in a novel mouse model of chronic traumatic encephalopathy. Surg. Neurol. Int. 5, 184

51. Lavin, C., Melis, C., Mikulan, E., Gelormini, C., Huepe, D., and Ibañez, A. (2013). The anterior cingulate cortex: an integrative hub for human socially-driven interactions. Front. Neurosci. 7, 64.

52. Tu, T.W., Williams, R.A., Lescher, J.D., Jikaria, N., Turtzo, L.C., and Frank, J.A. (2016). Radiological-pathological correlation of diffusion tensor and magnetization transfer imaging in a closed head traumatic brain injury model. Ann. Neurol. 79, 907-920.

53. Selwyn, R.G., Cooney, S.J., Khayrullina, G., Hockenbury, N., Wilson, C.M., Jaiswal, S., Bermudez, S., Armstrong, R.C., and Byrnes, K.R (2016). Outcome after repetitive mild traumatic brain injury is temporally related to glucose uptake profile at time of second injury. J. Neurotrauma 33, 1479-91

54. Meehan, W.P., 3rd, Zhang, J., Mannix, R. and Whalen, M.J. (2012). Increasing recovery time between injuries improves cognitive outcome after repetitive mild concussive brain injuries in mice. Neurosurgery $71,885-891$.

55. Mannix, R., Meehan, W.P., Mandeville, J., Grant, P.E., Gray, T., Berglass, J., Zhang, J., Bryant, J., Rezaie, S., Chung, J.Y., Peters, N.V., Lee, C., Tien, L.W., Kaplan, D.L., Feany, M., and Whalen, M. (2013). Clinical correlates in an experimental model of repetitive mild brain injury. Ann. Neurol. 74, 65-75.

56. Huang, L., Coats, J.S., Mohd-Yusof, A., Yin, Y., Assaad, S., Muellner, M.J., Kamper, J.E., Hartman, R.E., Dulcich, M., Donovan, V.M., Oyoyo, U., and Obenaus, A. (2013). Tissue vulnerability is increased following repetitive mild traumatic brain injury in the rat. Brain Res. 1499, 109-120.

57. Longhi, L., Saatman, K.E., Fujimoto, S., Raghupathi, R., Meaney, D.F., Davis, J., McMillan, B.S.A., Conte, V., Laurer, H.L., Stein, S., Stocchetti, N., and McIntosh, T.K. (2005). Temporal window of vulnerability to repetitive experimental concussive brain injury. Neurosurgery 56, 364-374.

58. Kou, Z and VandeVord, P.J. (2010). Traumatic whitle matter injury and glial activation: from basic science to clinics. Glia 62, 18311855
59. Jensen, J.H., and Helpern, J.A. (2010). MRI quantification of nonGaussian water diffusion by kurtosis analysis. NMR Biomed. 23, 698710.

60. Song, S.K., Yoshino, J., Le, T.Q., Lin, S.J., Sun, S.W., Cross, A.H., and Armstrong, R.C. (2005). Demyelination increases radial diffusivity in corpus callosum of mouse brain. NeuroImage 26 , 132-140.

61. Tobin, J.E., Xie, M., Le, T.Q., Song, S.K., and Armstrong, R.C. (2011). Reduced axonopathy and enhanced remyelination after chronic demyelination in fibroblast growth factor 2 (Fgf2)-null mice: differential detection with diffusion tensor imaging. J. Neuropathol. Exp. Neurol. 70, 157-165.

62. Alexander, A.L., Lee, J.E., Lazar, M., and Field, A.S. (2007). Diffusion tensor imaging of the brain. Neurotherapeutics 4, 316-329.

63. Singh, K., Trivedi, R., Devi, M.M., Tripathi, R.P., and Khushu, S (2016). Longitudinal changes in the DTI measures, anti-GFAP expression and levels of serum inflammatory cytokines following mild traumatic brain injury. Exp. Neurol. 275, 427-435.

64. Stemper, B.D., Shah, A.S., Pintar, F.A., McCrea, M., Kurpad, S.N., Glavaski-Joksimovic, A., Olsen, C., and Budde, M.D. (2015). Head rotational acceleration characteristics influence behavioral and diffusion tensor imaging outcomes following concussion. Ann. Biomed. Eng. 43, 1071-1088.

65. Meier, T.B., Bellgowan, P.S., Bergamino, M., Ling, J.M., and Mayer, A.R. (2016). Thinner cortex in collegiate football players with, but not without, a self-reported history of concussion. J. Neurotrauma 33, 330-338.

66. Zhou, Y., Kierans, A., Kenul, D., Ge, Y., Rath, J., Reaume, J., Grossman, R.I., and Lui, Y.W. (2013). Mild traumatic brain injury: longitudinal regional brain volume changes. Radiology 267, 880890.

67. McKee, A.C., Stern, R.A., Nowinski, C.J., Stein, T.D., Alvarez, V.E. Daneshvar, D.H., Lee, H.S., Wojtowicz, S.M., Hall, G., Baugh, C.M., Riley, D.O., Kubilus, C.A., Cormier, K.A., Jacobs, M.A., Martin, B.R., Abraham, C.R., Ikezu, T., Reichard, R.R., Wolozin, B.L., Budson, A.E., Goldstein, L.E., Kowall, N.W., and Cantu, R.C. (2013). The spectrum of disease in chronic traumatic encephalopathy. Brain $136,43-64$.

68. McKee, A.C., Cairns, N.J., Dickson, D.W., Folkerth, R.D., Keene, C.D., Litvan, I., Perl, D.P., Stein, T.D., Vonsattel, J.P., Stewart, W., Tripodis, Y., Crary, J.F., Bieniek, K.F., Dams-O'Connor, K., Alvarez, V.E., and Gordon, W.A.; TBI/CTE group. (2016). The first NINDS/ NIBIB consensus meeting to define neuropathological criteria for the diagnosis of chronic traumatic encephalopathy. Acta Neuropathol. $131,75-86$.

69. Srivastava, D.P., Jones, K.A., Woolfrey, K.M., Burgdorf, J., Russell, T.A., Kalmbach, A., Lee, H., Yang, C., Bradberry, M.M., Wokosin, D., Moskal, J.R., Casanova, M.F., Waters, J., and Penzes, P. (2012). Social, communication, and cortical structural impairments in Epac2 deficient mice. J. Neurosci. 32, 11864-11878.

70. Silverman, J.L., Yang, M., Lord, C., and Crawley, J.N. (2010). Behavioural phenotyping assays for mouse models of autism. Nat. Rev. Neurosci. 11, 490-502.

71. Nadler, J.J., Moy, S.S., Dold, G., Trang, D., Simmons, N., Perez, A., Young, N.B., Barbaro, R.P., Piven, J., Magnuson, T.R., and Crawley, J.N. (2004). Automated apparatus for quantitation of social approach behaviors in mice. Genes Brain Behav. 3, 303-314.

72. Klemenhagen, K.C., O'Brien, S.P., and Brody, D.L. (2013). Repetitive concussive traumatic brain injury interacts with post-injury foot shock stress to worsen social and depression-like behavior in mice. PLoS One 8, e74510.

73. Bennett, R.E., Mac Donald, C.L., and Brody, D.L. (2012). Diffusion tensor imaging detects axonal injury in a mouse model of repetitive closed-skull traumatic brain injury. Neurosci. Lett. 513, $160-165$

74. Hernandez, A., Donovan, V., Grinberg, Y.Y., Obenaus, A., and Carson, M.J. (2016). Differential detection of impact site versus rotational site injury by magnetic resonance imaging and microglial morphology in an unrestrained mild closed head injury model. J. Neurochem. 136, $18-28$.

75. Robinson, S., Berglass, J.B., Denson, J.L., Berkner, J., Anstine, C.V., Winer, J.L., Maxwell, J.R., Qiu, J., Yang, Y., Sillerud, L.O., Meehan, W.P. 3rd, Mannix, R., and Jantzie, L.L. (2016). Microstructural and microglial changes after repetitive mild traumatic brain injury in mice. 
J. Neurosci. Res. 2016 Jul 25. doi: 10.1002/jnr.23848. [Epub ahead of print]

76. Hong, S., Dissing-Olesen, L., and Stevens, B. (2016). New insights on the role of microglia in synaptic pruning in health and disease. Curr. Opin. Neurobiol. 36, 128-134.

77. Sekar, A., Bialas, A.R., de Rivera, H., Davis, A., Hammond, T.R., Kamitaki, N., Tooley, K., Presumey, J., Baum, M., Van Doren, V., Genovese, G., Rose, S.A., Handsaker, R.E., Schizophrenia Working Group of the Psychiatric Genomics, C., Daly, M.J., Carroll, M.C., Stevens, B., and McCarroll, S.A. (2016). Schizophrenia risk from complex variation of complement component 4 . Nature 530, $177-183$.

78. Olmos-Alonso, A., Schetters, S.T., Sri, S., Askew, K., Mancuso, R., Vargas-Caballero, M., Holscher, C., Perry, V.H., and Gomez-Nicola,
D. (2016). Pharmacological targeting of CSF1R inhibits microglial proliferation and prevents the progression of Alzheimer's-like pathology. Brain 139, 891-907.

Address correspondence to: Fengshan $Y u, M D$ Center for Neuroscience and Regenerative Medicine Department of Anatomy, Physiology and Genetics Uniformed Services University of the Health Sciences 4301 Jones Bridge Road Bethesda, MD 20814

E-mail: fengshan.yu.ctr@usuhs.edu 